\title{
Fear of Falling in Older Adults: A Scoping Review of Recent Literature
}

\author{
Scott MacKay, BSc Kin ${ }^{1}$, Patricia Ebert, PhD, RPsych ${ }^{2,3,4}$, Cathy Harbidge, BScPT ${ }^{4}$, \\ David B. Hogan, MD, FCPSC 5 \\ ${ }^{1}$ Department of Medicine, Cumming School of Medicine, University of Calgary, Calgary; ${ }^{2}$ Department of Psychology, \\ University of Calgary, Calgary; ${ }^{3}$ Hotchkiss Brain Institute, University of Calgary, Calgary; ${ }^{4}$ Specialized Geriatric Services, \\ Calgary Regional Health Authority, Calgary; ${ }^{5}$ Division of Geriatric Medicine, Department of Medicine, Cumming School \\ of Medicine, University of Calgary, Calgary, $A B$
}

https://doi.org/10.5770/cgj.24.521

\begin{abstract}
\section{Background}

Fear of falling (FOF) is prevalent among older adults and associated with adverse health outcomes. Over recent years a substantial body of research has emerged on its epidemiology, associated factors, and consequences. This scoping review summarizes the FOF literature published between April 2015 and March 2020 in order to inform current practice and identify gaps in the literature.
\end{abstract}

\section{Methods}

A total of 439 articles related to FOF in older adults were identified, 56 selected for full-text review, and 46 retained for data extraction and synthesis.

\section{Results}

The majority of included studies were cross-sectional. Older age, female sex, previous falls, worse physical performance, and depressive symptoms were the factors most consistently associated with FOF. Studies that measured FOF with a single question reported a significantly lower prevalence of FOF than those using the Falls Efficacy Scale, a continuous measure. FOF was associated with higher likelihoods of future falls, short-term mortality, and functional decline.

\section{Conclusions}

Comparisons between studies were limited by inconsistent definition and measurement of FOF, falls, and other characteristics. Consensus on how to measure FOF and which participant characteristics to evaluate would address this issue. Gaps in the literature include clarifying the relationships between FOF and cognitive, psychological, social, and environmental factors.

Key words: fear of falling, falls, older adults, scoping review

\section{INTRODUCTION}

In geriatric practice, fear of falling (FOF) was initially defined as a phobic reaction to standing or walking called "ptophobia". (1) The concept of FOF has since expanded to include reductions in balance self-efficacy, ${ }^{(2,3)}$ fearful anticipation of falling, ${ }^{(4)}$ and/or a deleterious avoidance of activity resulting from FOF. ${ }^{(5)}$ Prevalence estimates among older persons for FOF range from 20 to $39 \%$ overall $^{(6)}$ and from 40 to $73 \%$ in those who have fallen. ${ }^{(7)}$ The consequences of FOF for older persons include loss of independence, deconditioning from activity restriction, greater risk for subsequent falls, reduced social activity, and lower quality of life. ${ }^{(6,8)}$

Given its high prevalence and harmful consequences, there is a substantial body of research on identifying factors associated with FOF. Risk factors for FOF include sociodemographic, physical, psychological, and environmental factors. ${ }^{(7-10)} \mathrm{FOF}$ has also been studied as a predictor of future falls ${ }^{(11)}$ and declines in cognition, ${ }^{(12)}$ function, ${ }^{(13)}$ and shortterm survival. ${ }^{(14)}$ Considerably less work has been done recently to synthesize these emerging findings to inform current practice and future research related to FOF.

Current FOF measures range from asking a single question about FOF (e.g., "Are you afraid of falling?") to scales examining either FOF during specific activities (e.g., Survey of Activities and Fear of Falling in the Elderly ${ }^{(15)}$ ) or perceived self-efficacy in one's balance and/or ability to avoid falls (e.g., Falls Efficacy Scale ${ }^{(16)}$ ). It is unclear what effect these different operational definitions have on the detection and prevalence of FOF in older populations. The lack of a consistent definition for FOF has led to making comparisons between studies difficult. ${ }^{(7,17)}$

The aim of this scoping review is to examine broad aspects of the FOF literature, including the world-wide epidemiology of FOF, the different approaches used to detect FOF and their impact on prevalence estimates, factors associated with its occurrence and outcomes, and knowledge 
gaps that future research could address. The most recent systematic review of factors associated with FOF was by Denkinger et al. ${ }^{(18)}$ It was published in 2015. Our intent was also to update this review of FOF and the factors associated with it by examining publications published since 2015. To address these objectives, we performed a scoping review of observational studies on FOF published from April 2015 to March 2020. This methodology ${ }^{(19)}$ was chosen as it best aligned with our desire to: (1) summarize recent FOF research; and, (2) use this data to inform current practice and the need for future research on FOF.

\section{METHODS}

\section{Initial Search Strategy}

Using the free text term "fear of falling" in late April of 2020, we searched for relevant articles on the PubMed database that were published between April 1, 2015 and March 31, 2020. This search was restricted to publications in English that included adults $\geq 65$ years of age. PubMed was utilized as we were interested in published biomedical and life sciences findings.

\section{Selection Process}

The selection of papers took place in two steps. First, two independent reviewers (SM, DBH) reviewed all titles and abstracts to select papers for full-text review. Studies were excluded if they did not represent original research (note: we did include any scoping or systematic reviews on FOF published during the time period of interest); were restricted to a specific disease or clinic population (e.g., patients with cerebrovascular disease); dealt solely with interventions for FOF (we wished to avoid confounding by the impact of the intervention); reported solely on associations with performance-based measures (e.g., gait speed); used only qualitative methods to assess FOF; assessed only the impact of FOF on a third party (e.g., caregiver); and/or, sampled only older adults not residing in the community (e.g., institutionalized individuals). Included studies had to: measure and report on FOF; perform quantitative analyses involving FOF (e.g., examining an association between FOF and some other variable); have a study population with a mean participant age $\geq 60$; and, not meet any of the exclusionary criteria. Duplicate publications were removed at this stage. Discordant ratings were discussed by the two raters with a final consensus decision reached.

In the second step, full texts of selected articles were retrieved and reviewed using the same criteria by two pairs of independent reviewers. One pair ( $\mathrm{CH}$ and $\mathrm{PE}$ ) reviewed articles where the last initial of the lead authors were A-H while the other pair (SM and DBH) reviewed papers where the last initial of the lead authors were I-Z. Papers could be excluded at this stage based on the noted inclusion or exclusion criteria. Any discordant ratings as to whether the paper should be retained were discussed by the two raters with a final consensus decision reached. Articles retained after this review went on to the data extraction and synthesis stage.

\section{Data Extraction}

Using a standardized data extraction form, the same two pairs of independent reviewers examined their assigned articles and extracted data on: study population; approach used to detect FOF; prevalence and/or incidence of FOF; factors associated with FOF; and the effect of FOF on all relevant outcomes reported. Data extraction also included comments on study limitations and potential sources of bias present in the selected articles. Any discrepancies in the data extracted were dealt with by review of the source paper. These findings were then collated and analyzed by all the authors with consensus reached on the interpretation of the data and the conclusions reached in this review.

\section{Statistical Analyses}

Descriptive and inferential statistics were extracted from the papers reviewed. De novo statistical analyses were conducted using IBM SPSS version 24.0 (IBM, Armonk, NY, USA). An independent samples $t$-test was used to compare mean FOF prevalence values between studies that used single questions compared to those that used the FES to measure FOF. This analysis was two-tailed with significance set at $p<.05$.

\section{RESULTS}

\section{Search Results}

A total of 439 articles related to FOF in older adults were identified. Fifty-six were selected by at least one reviewer for full-text review. Agreement on the title and abstract screening was $97.7 \%$. The four articles with discordant results were excluded after discussion. An additional five were excluded at the full-text review stage and one was excluded during data extraction. Forty-six articles were retained for data extraction and synthesis (Table 1). Figure 1 shows a flow diagram of the selection process.

\section{Study Design}

The majority of studies (30/46, 65.2\%) used a cross-sectional study design. Longitudinal study designs (14/46, 30.4\%) were the next most common approach. These longitudinal studies ranged from one ${ }^{(20)}$ to 10 years ${ }^{(21)}$ in duration. Two systematic reviews $(4.3 \%)$ were also included. ${ }^{(22,23)}$

Included studies took place in whole or in part within 20 countries/regions and four continents (Asia, Europe, and both North and South America). The specific locations (listed alphabetically) and the number of studies that took place in each were: Albania (3), Brazil (8), Canada (4), Colombia (3), Europe (2), Germany (1), Hong Kong (1), Ireland (3), Israel (2), Netherlands (1), Norway (1), Portugal (1), Serbia (1), South Korea (6), Spain (2), Sweden (1), Taiwan (2), United Kingdom(1), USA (7), and Vietnam (1). Five studies included participants from multiple countries/regions. ${ }^{(11,13,24-26)}$ The two systematic reviews ${ }^{(22,23)}$ included articles from multiple countries. FOF was commonly found in older populations across the world. In studies that used a single question for detection, its prevalence ranged from $25.89 \%$ (SD 10.08) 
MACKAY: FEAR OF FALLING IN OLDER ADULTS

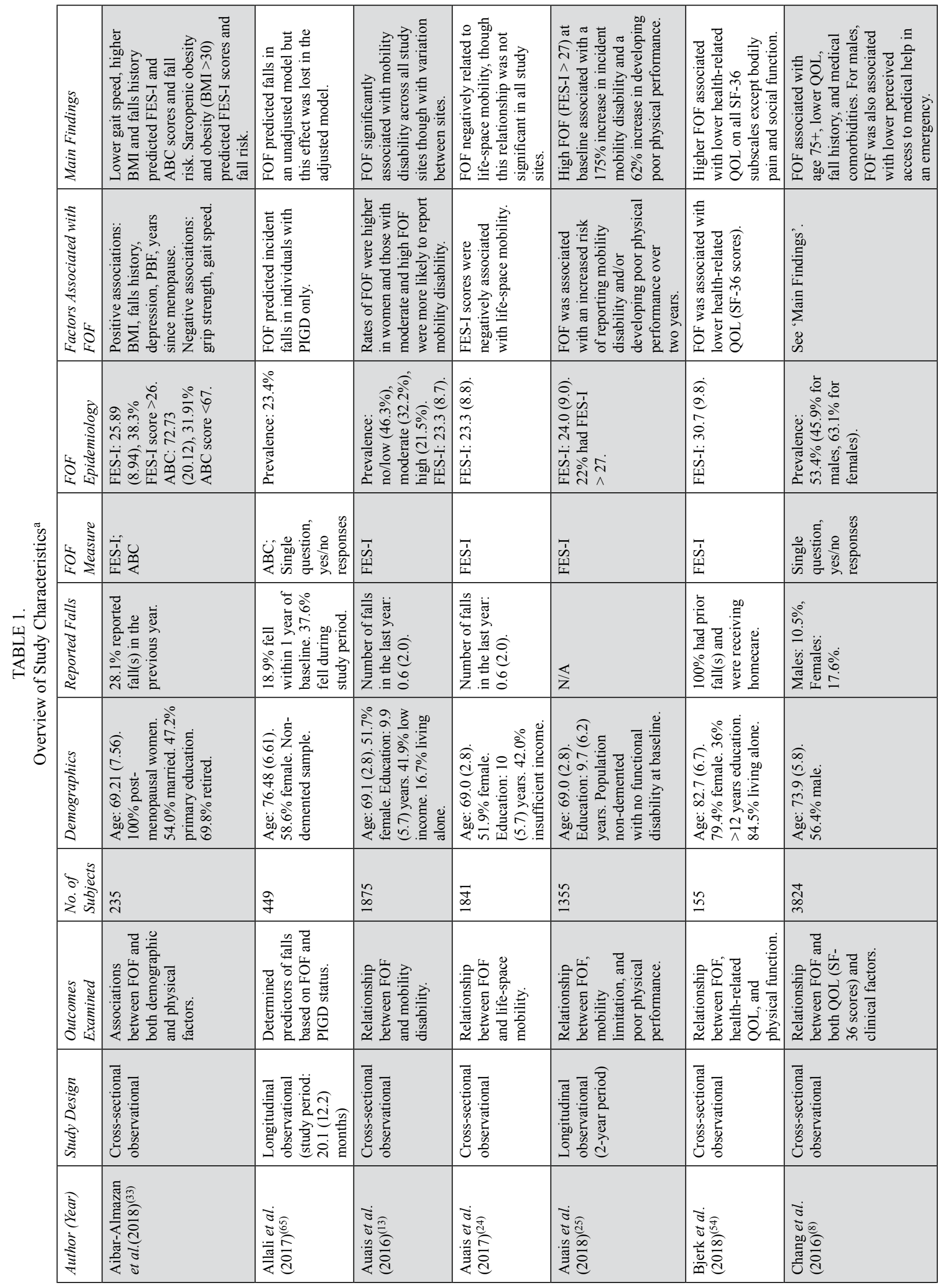




\begin{tabular}{|c|c|c|c|c|c|c|}
\hline 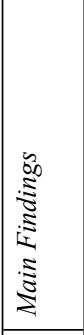 & 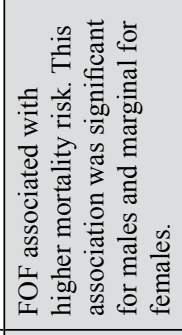 & 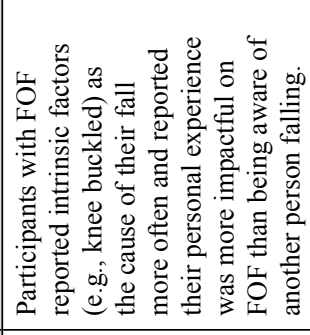 & 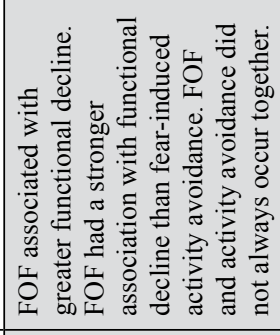 & 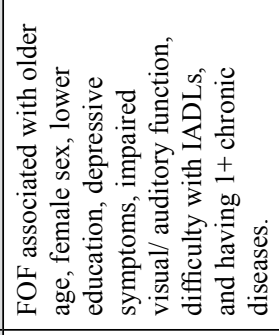 & 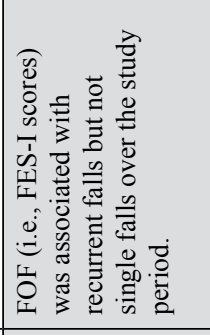 & 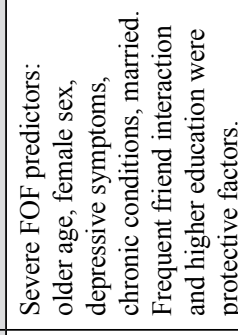 \\
\hline 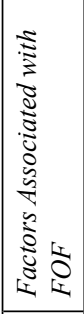 & 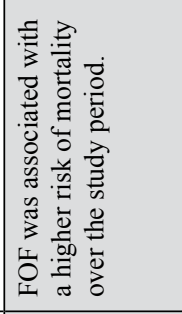 & 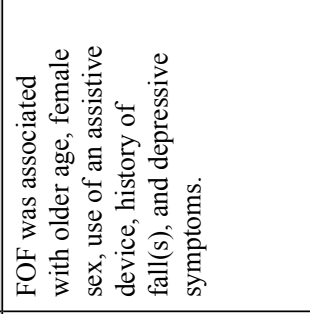 & 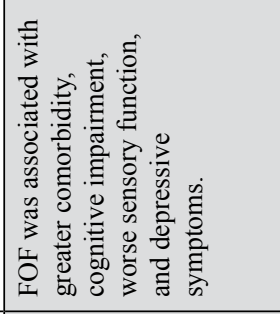 & 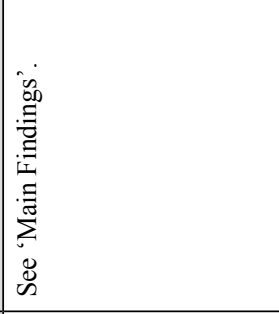 & 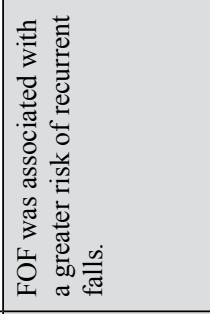 & 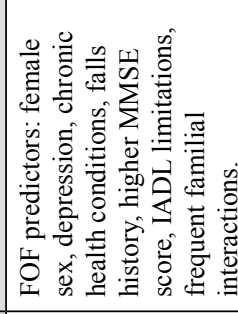 \\
\hline 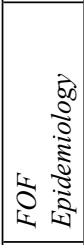 & 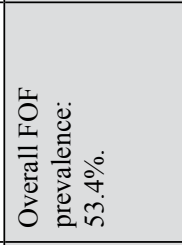 & 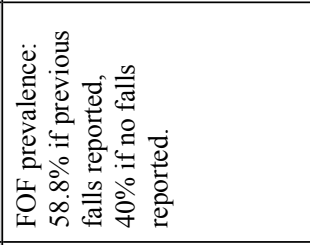 & 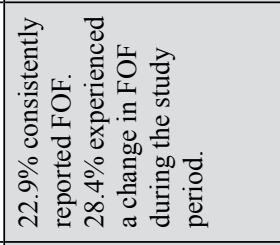 & 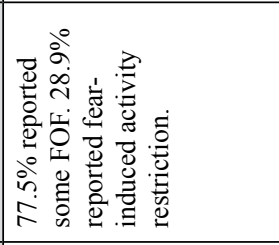 & 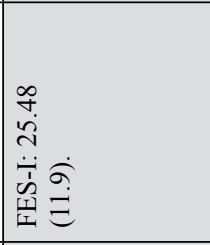 & 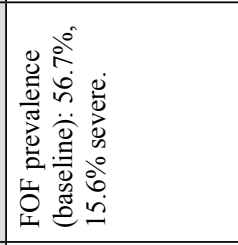 \\
\hline 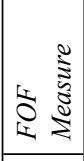 & 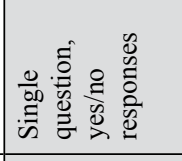 & 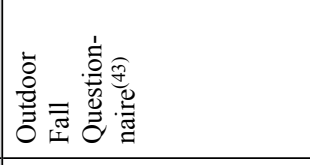 & 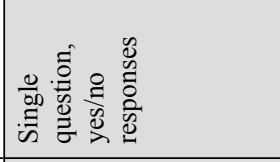 & 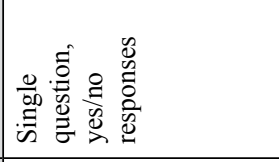 & \begin{tabular}{|l}
$\vec{s}$ \\
空 \\
\end{tabular} & 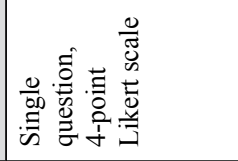 \\
\hline 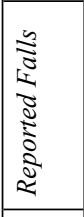 & 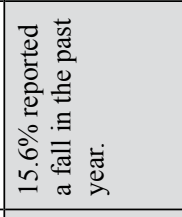 & 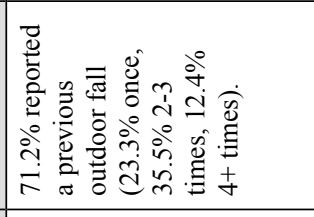 & 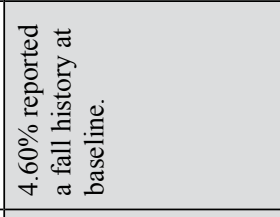 & 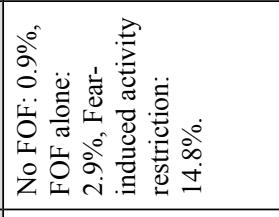 & 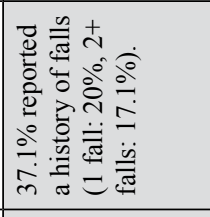 & 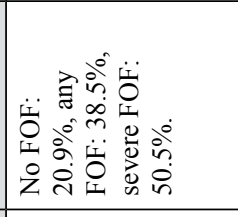 \\
\hline 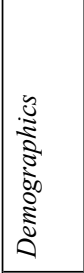 & 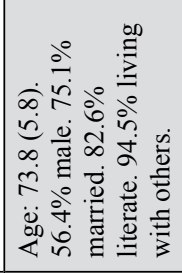 & 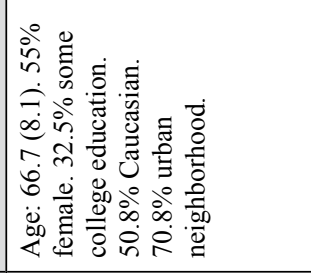 & 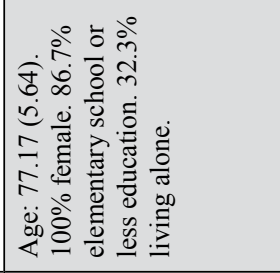 & 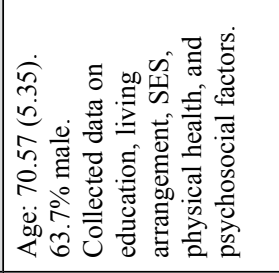 & 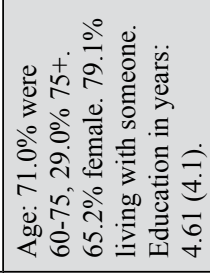 & 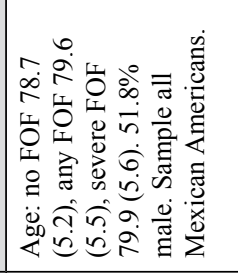 \\
\hline 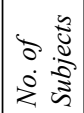 & $\underset{\substack{\infty \\
\infty}}{\stackrel{\Delta}{\infty}}$ & 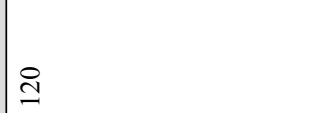 & : & 竎 & 年 & 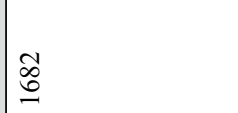 \\
\hline 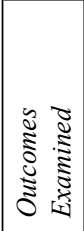 & 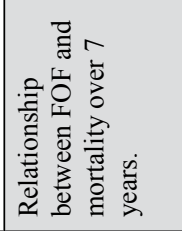 & 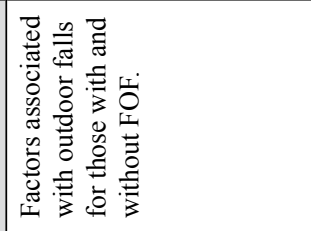 & 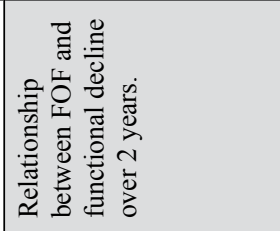 & 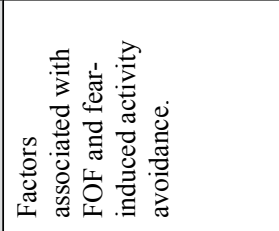 & 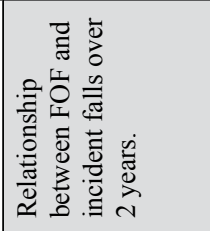 & 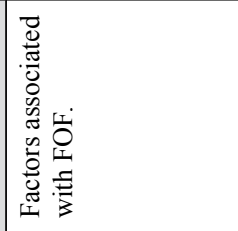 \\
\hline 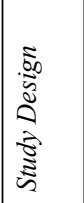 & 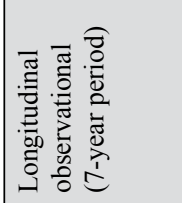 & 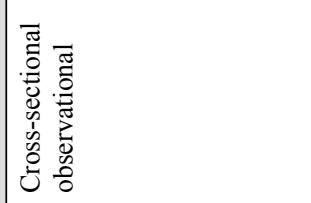 & 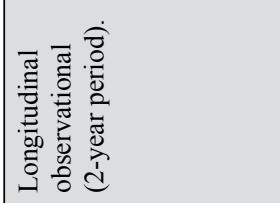 & 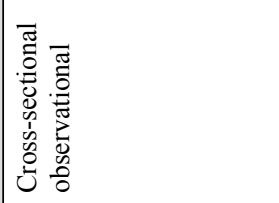 & 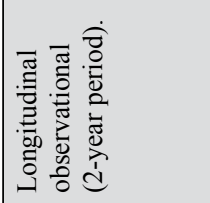 & 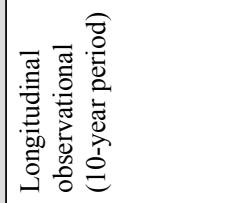 \\
\hline 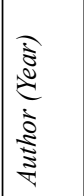 & 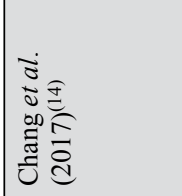 & 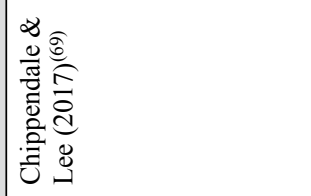 & 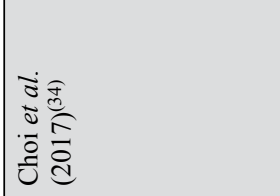 & 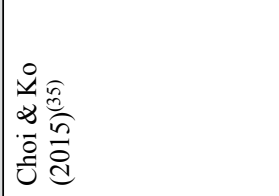 & 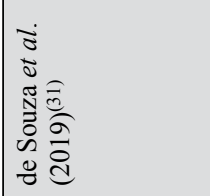 & 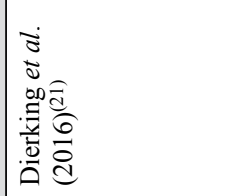 \\
\hline
\end{tabular}


MACKAY: FEAR OF FALLING IN OLDER ADULTS

\begin{tabular}{|c|c|c|c|c|c|}
\hline 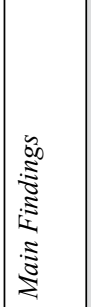 & 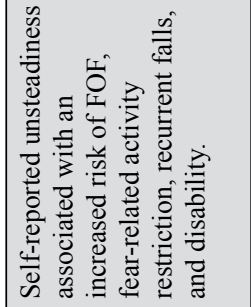 & 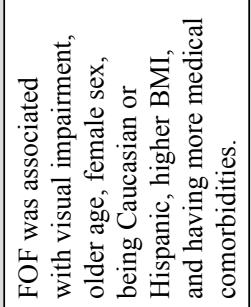 & 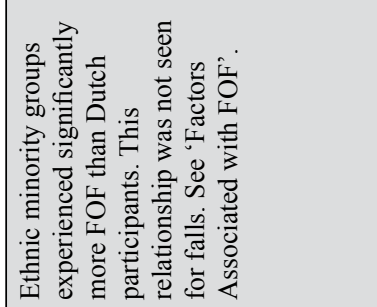 & 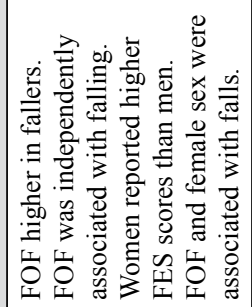 & 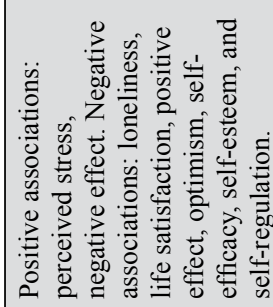 \\
\hline 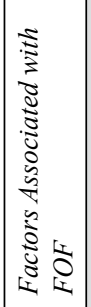 & 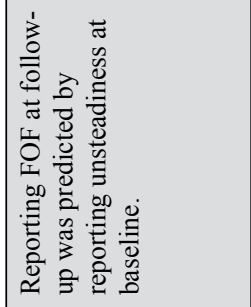 & 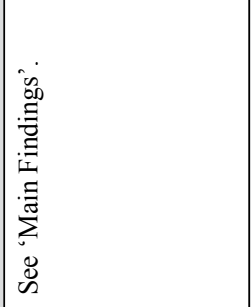 & 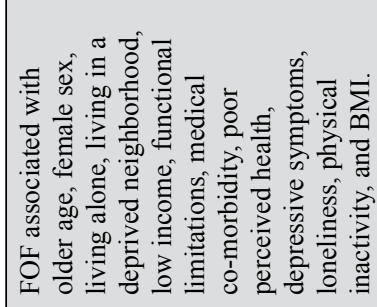 & 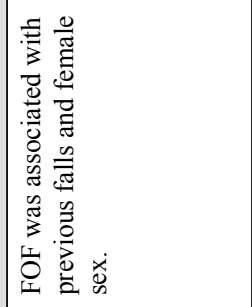 & 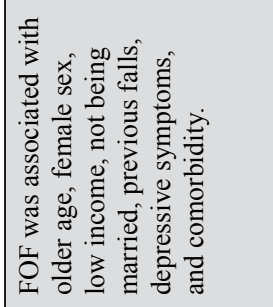 \\
\hline 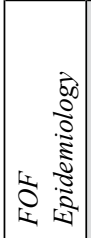 & 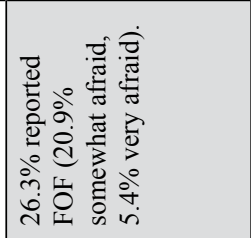 & 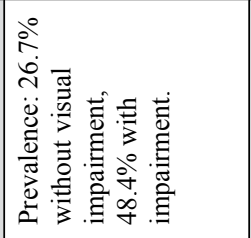 & 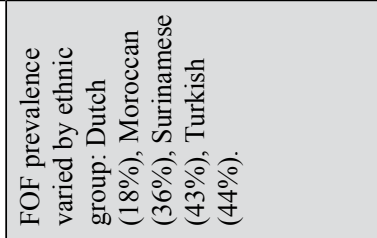 & 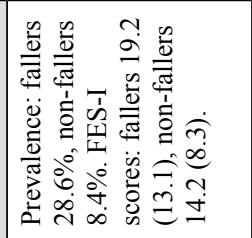 & 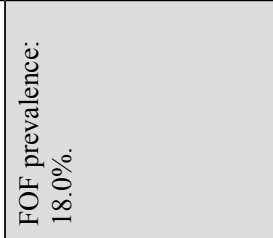 \\
\hline 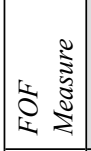 & 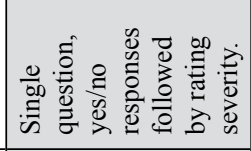 & 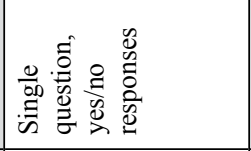 & 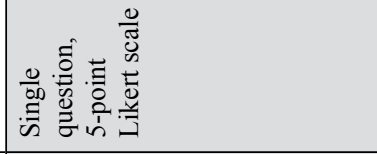 & 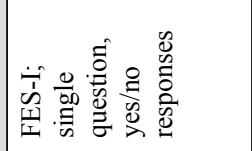 & 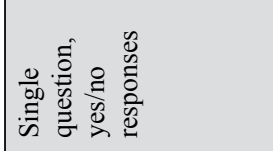 \\
\hline 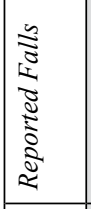 & 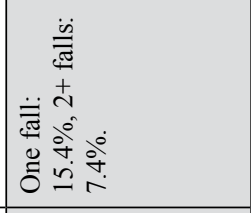 & 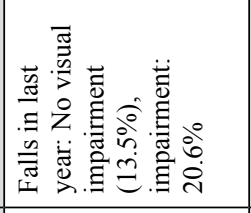 & 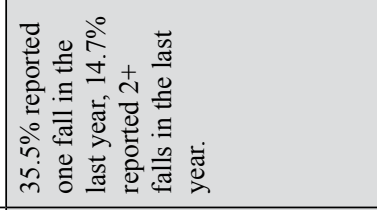 & 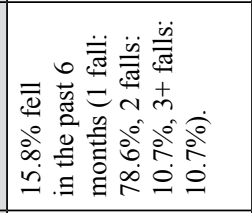 & $\overleftrightarrow{z}$ \\
\hline 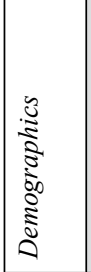 & 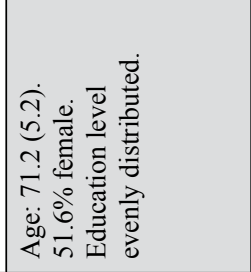 & 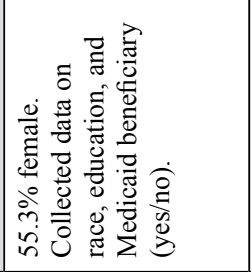 & 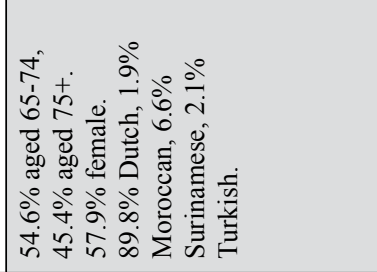 & 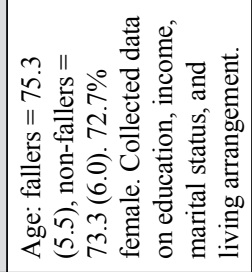 & 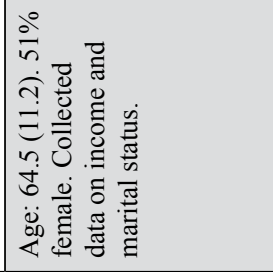 \\
\hline 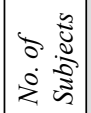 & $\overrightarrow{\widetilde{\sigma}}$ & $\begin{array}{l}\infty \\
\stackrel{n}{\varrho} \\
=\end{array}$ & $\begin{array}{l}\widetilde{\infty} \\
\infty \\
\infty\end{array}$ & 芯 & 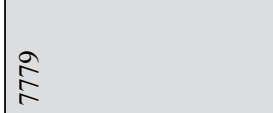 \\
\hline 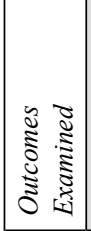 & 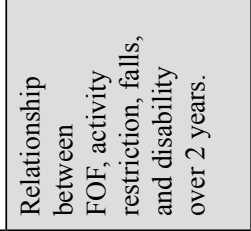 & 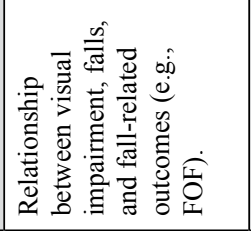 & 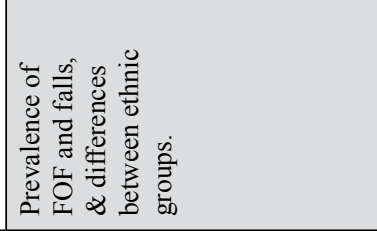 & 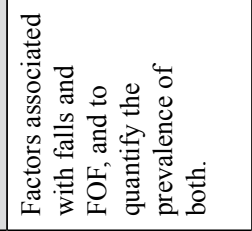 & 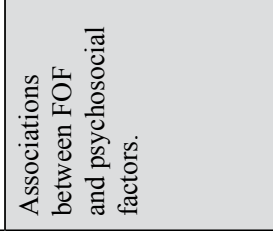 \\
\hline 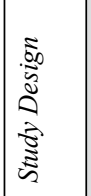 & 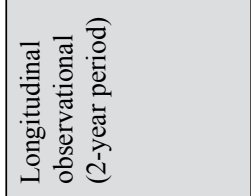 & 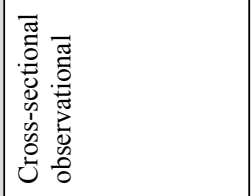 & 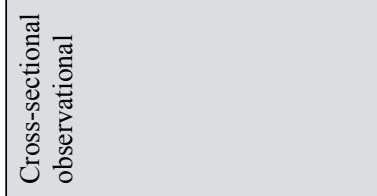 & 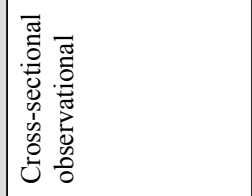 & 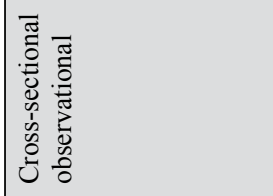 \\
\hline 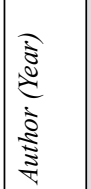 & 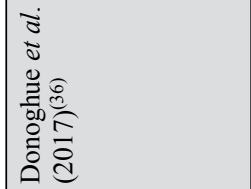 & 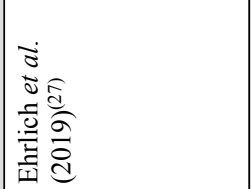 & 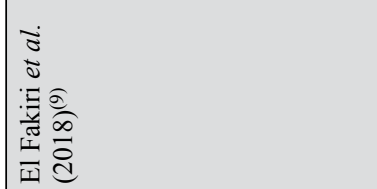 & 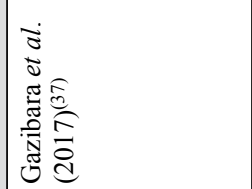 & 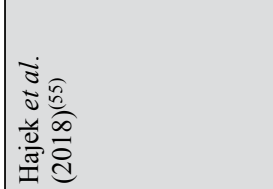 \\
\hline
\end{tabular}




\begin{tabular}{|c|c|c|c|c|c|}
\hline 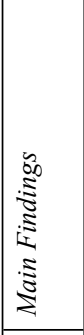 & 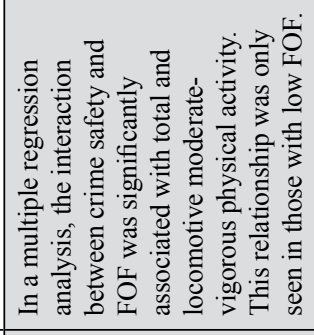 & 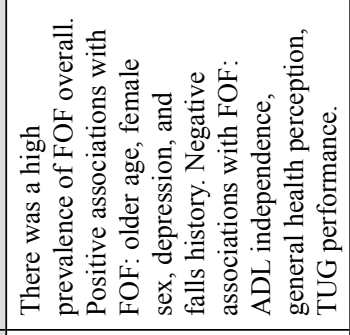 & 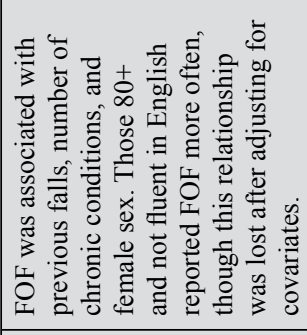 & 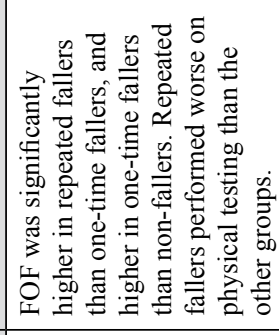 & 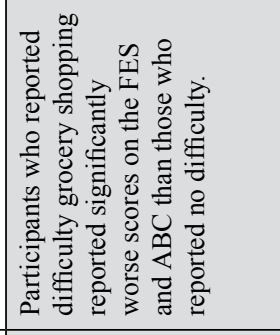 \\
\hline 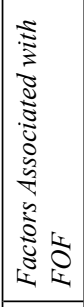 & 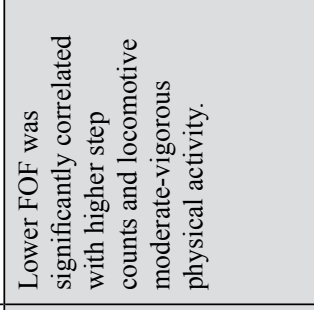 & 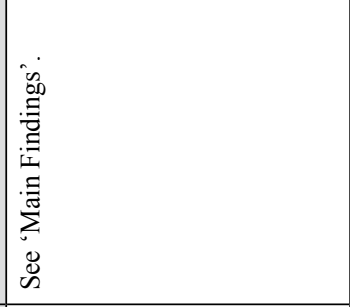 & 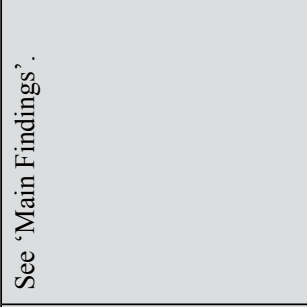 & 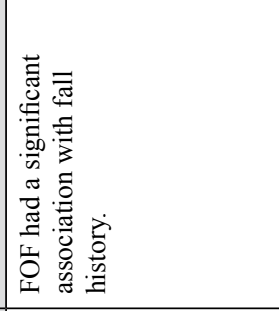 & 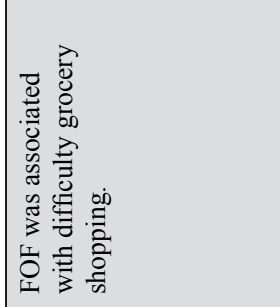 \\
\hline 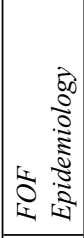 & 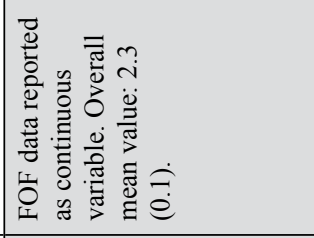 & 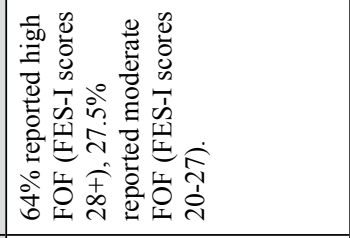 & 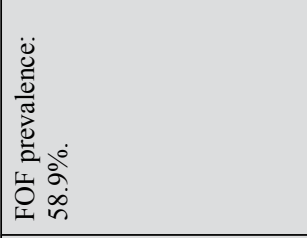 & 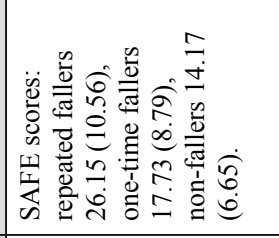 & 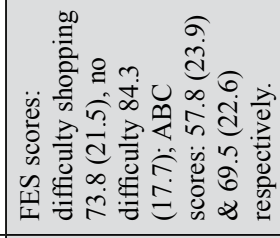 \\
\hline 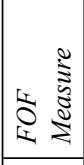 & 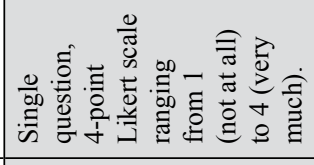 & \begin{tabular}{|l}
$\overrightarrow{1}$ \\
鲁
\end{tabular} & 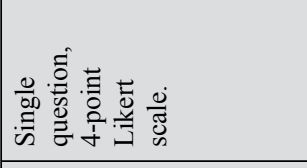 & 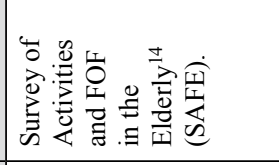 & 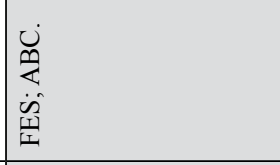 \\
\hline 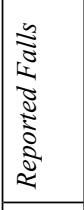 & 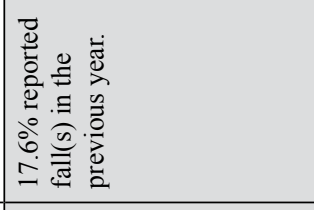 & 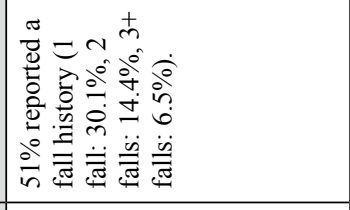 & 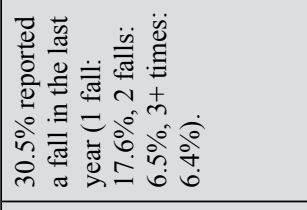 & 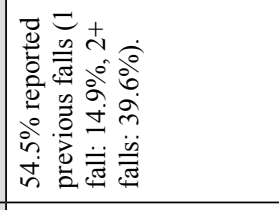 & 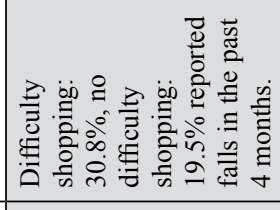 \\
\hline 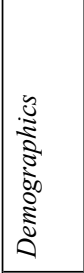 & 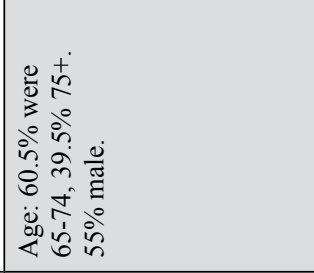 & 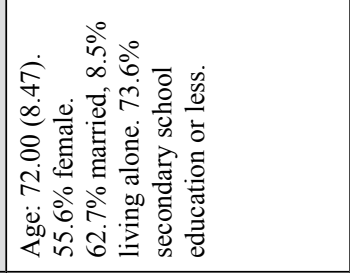 & 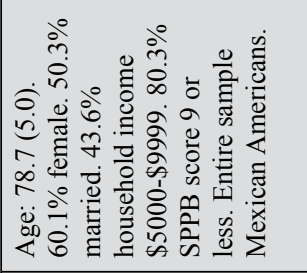 & 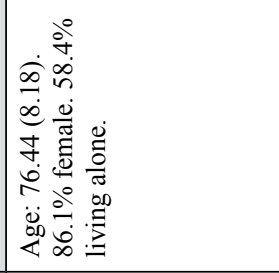 & 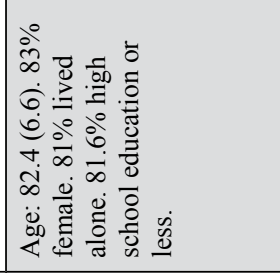 \\
\hline 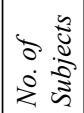 & $\underset{\sim}{\infty}$ & $\tilde{\Omega}$ & $\stackrel{i}{=}$ & 흐 & $\propto$ \\
\hline 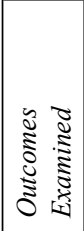 & 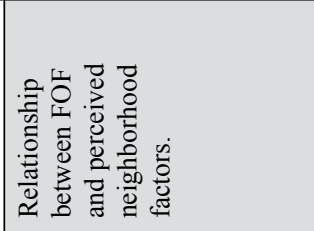 & 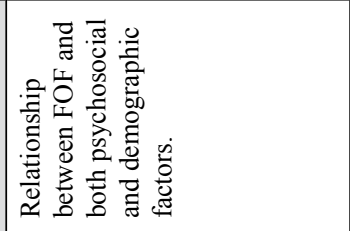 & 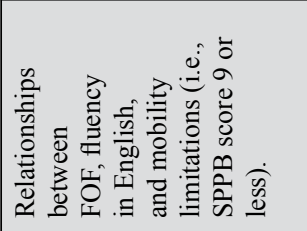 & 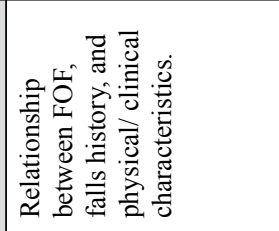 & 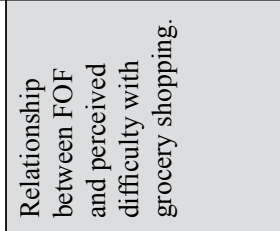 \\
\hline 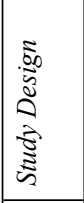 & 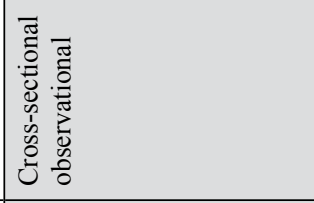 & 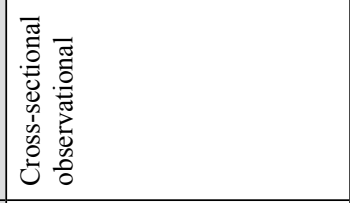 & 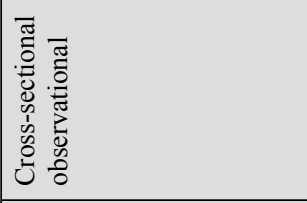 & 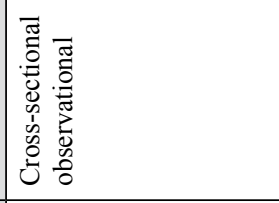 & 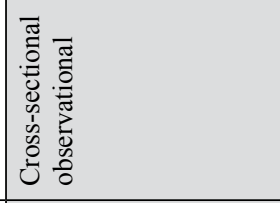 \\
\hline 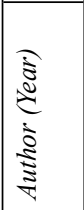 & 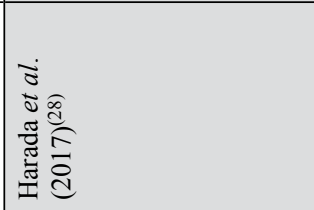 & 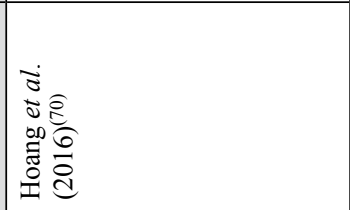 & 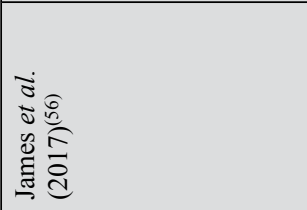 & 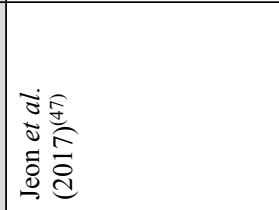 & 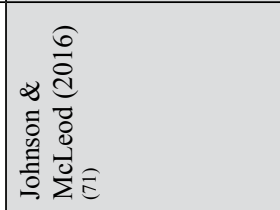 \\
\hline
\end{tabular}


MACKAY: FEAR OF FALLING IN OLDER ADULTS

\begin{tabular}{|c|c|c|c|c|c|c|}
\hline 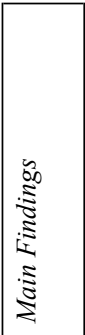 & 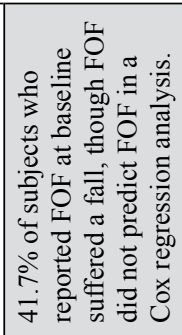 & 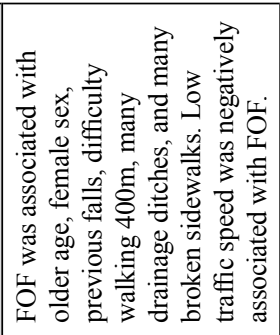 & 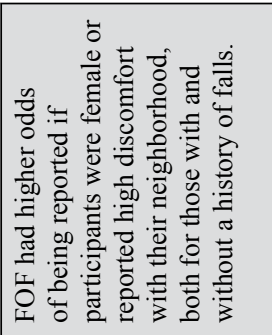 & 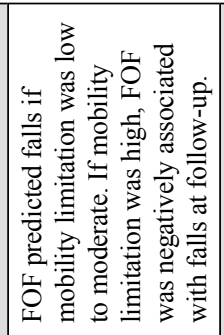 & 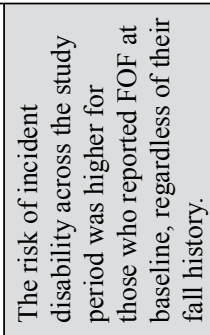 & 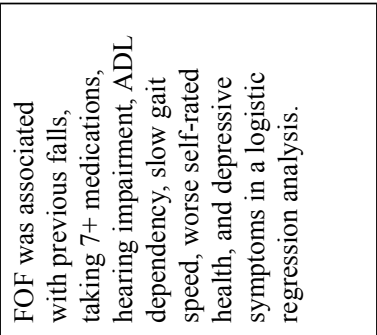 \\
\hline 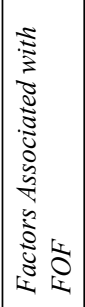 & 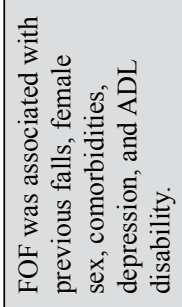 & 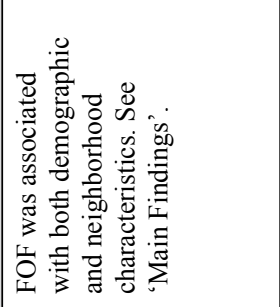 & 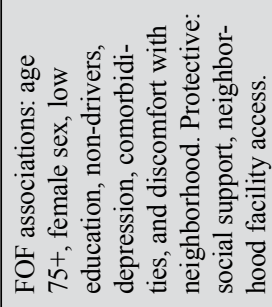 & 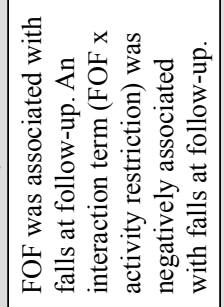 & 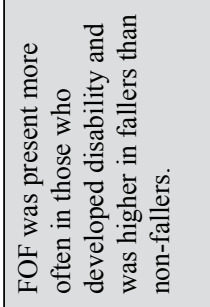 & 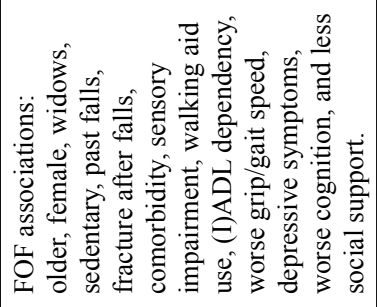 \\
\hline 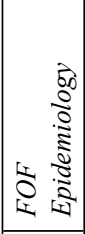 & 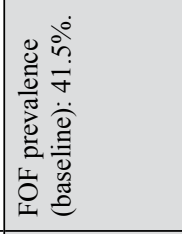 & 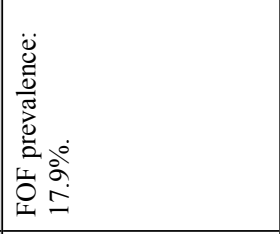 & 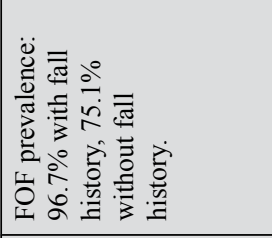 & 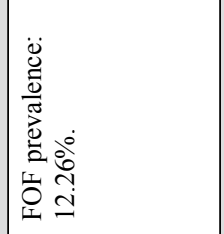 & 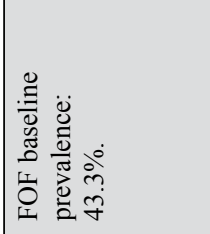 & 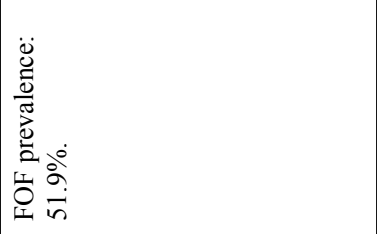 \\
\hline 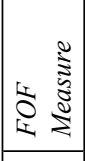 & 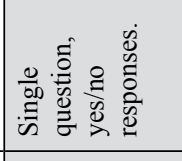 & 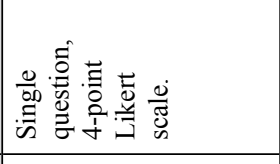 & 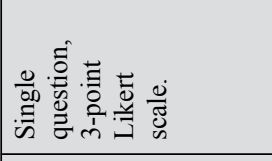 & 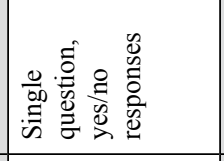 & 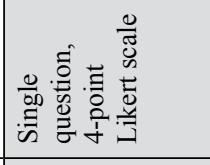 & 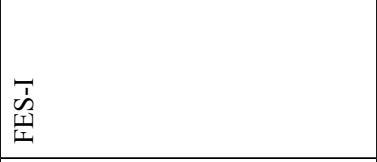 \\
\hline 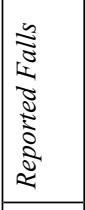 & 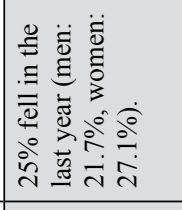 & 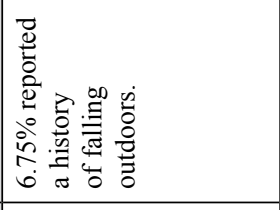 & 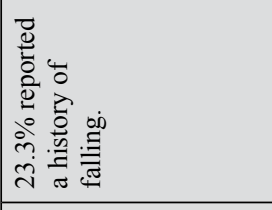 & 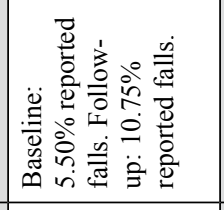 & 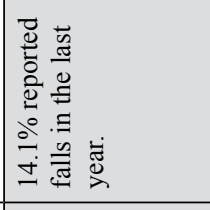 & 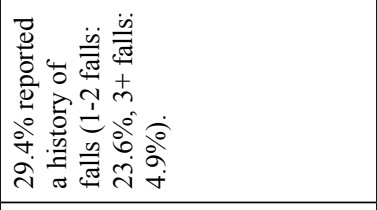 \\
\hline 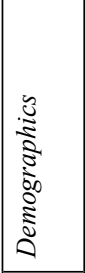 & 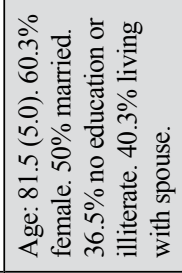 & 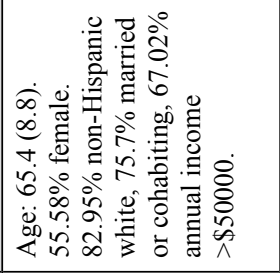 & 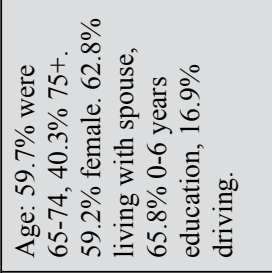 & 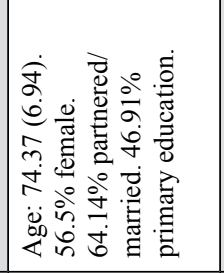 & 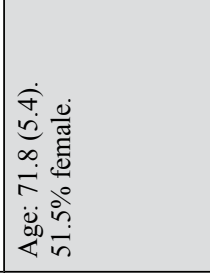 & 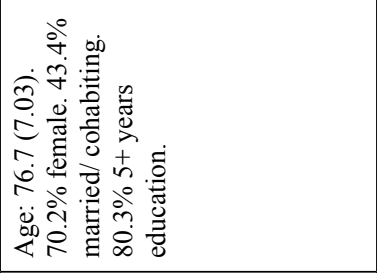 \\
\hline 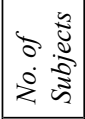 & 过 & 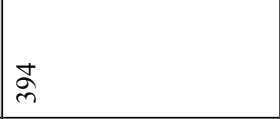 & 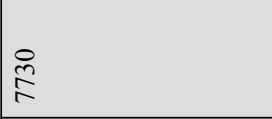 & 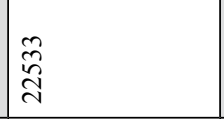 & ซे & I \\
\hline 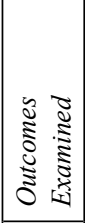 & 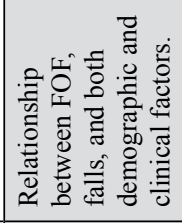 & 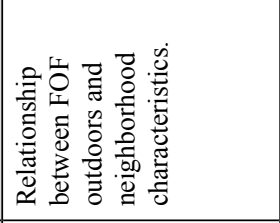 & 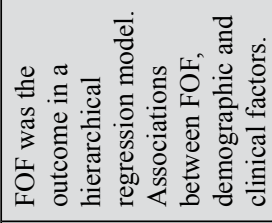 & 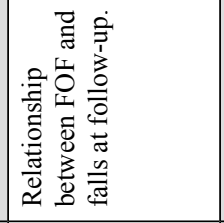 & 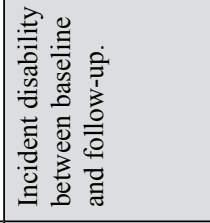 & 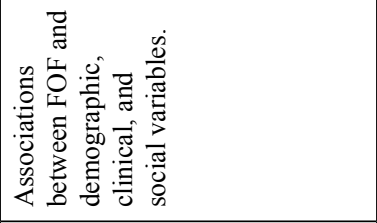 \\
\hline 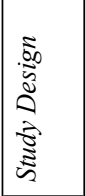 & 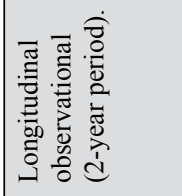 & 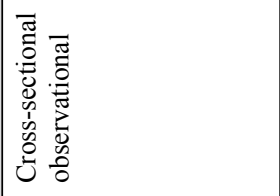 & 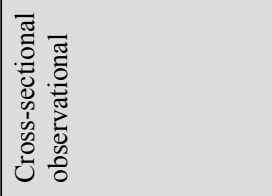 & 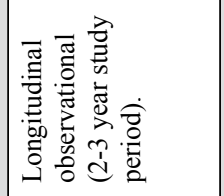 & 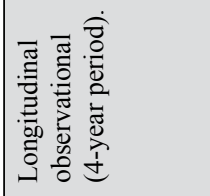 & 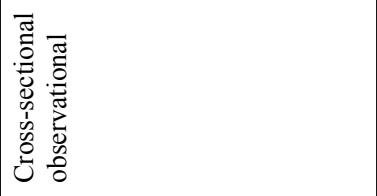 \\
\hline 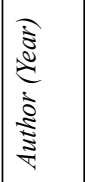 & 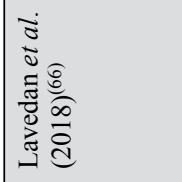 & 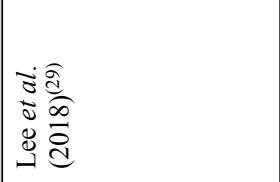 & 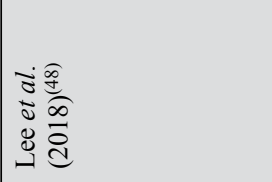 & 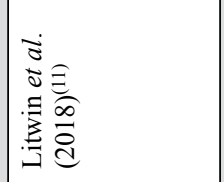 & 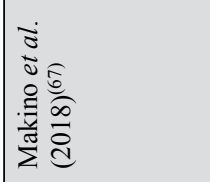 & 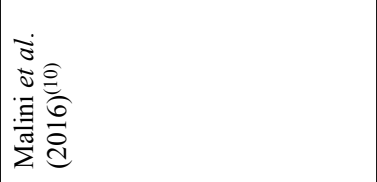 \\
\hline
\end{tabular}




\begin{tabular}{|c|c|c|c|c|c|}
\hline 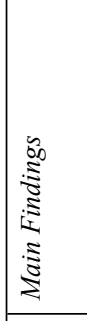 & 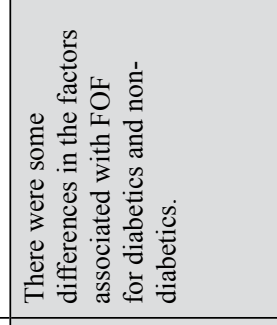 & 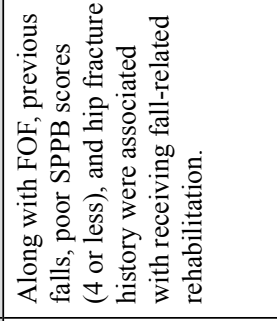 & 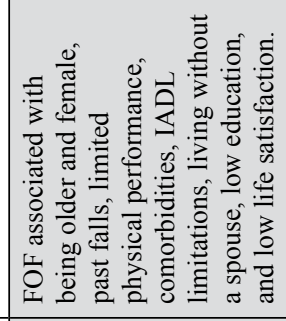 & 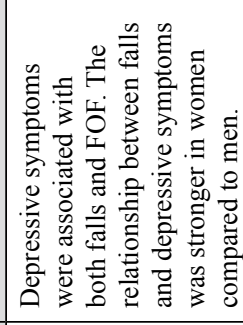 & 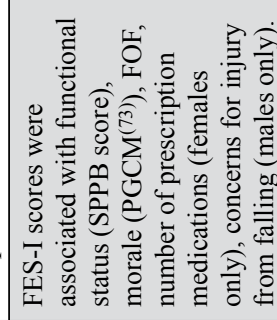 \\
\hline 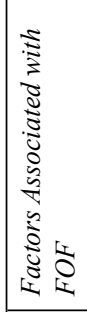 & 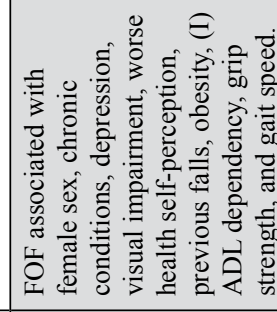 & 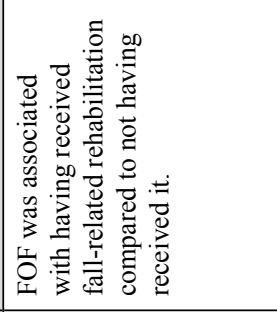 & 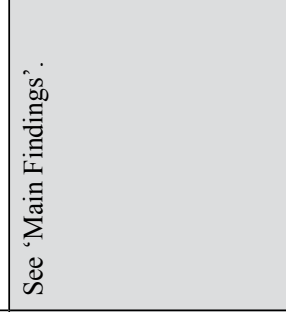 & 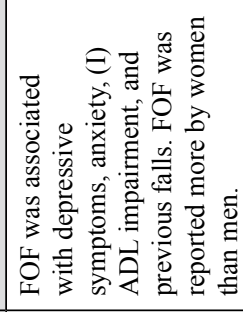 & 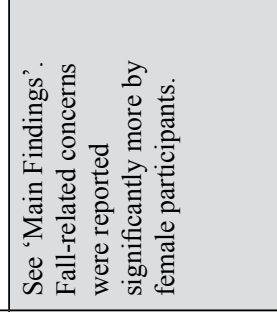 \\
\hline 离 & 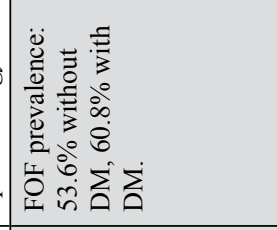 & 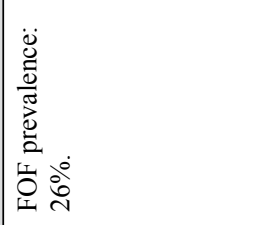 & 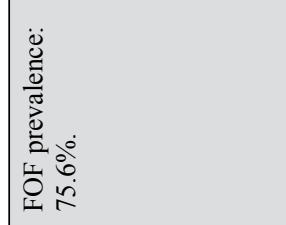 & 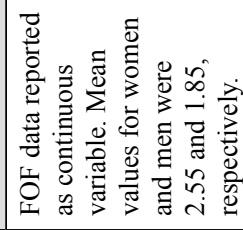 & 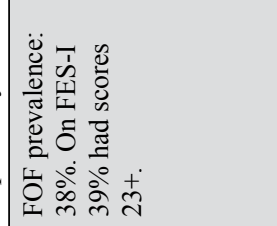 \\
\hline 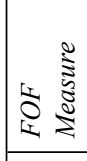 & 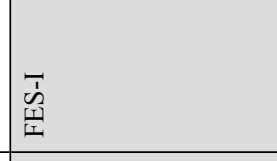 & 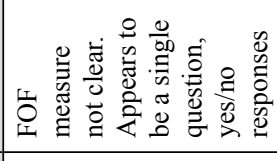 & 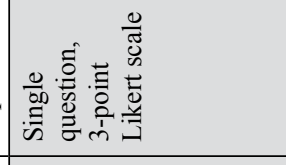 & 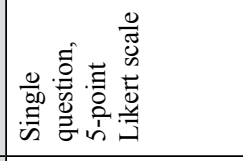 & 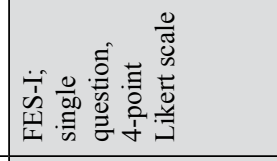 \\
\hline 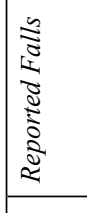 & 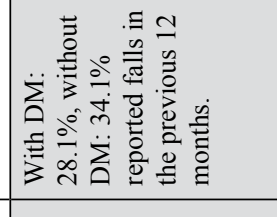 & 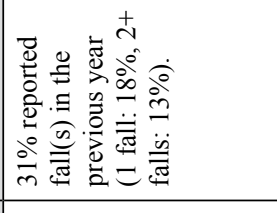 & 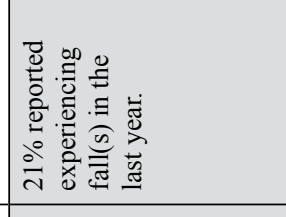 & 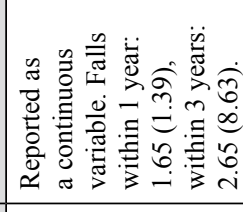 & 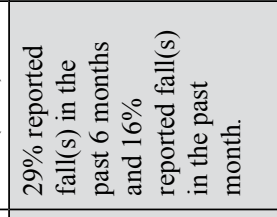 \\
\hline 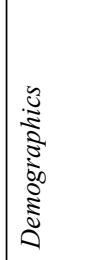 & 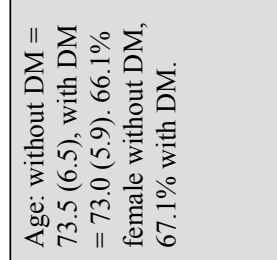 & 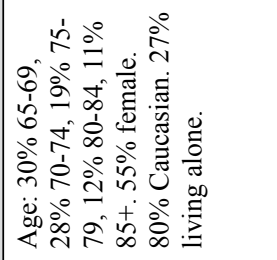 & 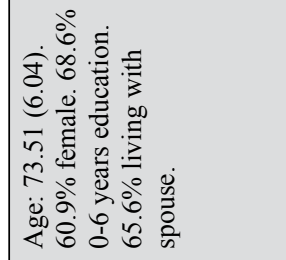 & 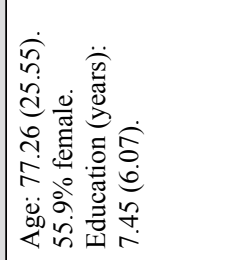 & 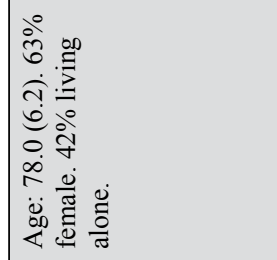 \\
\hline 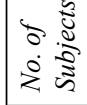 & 㠻 & 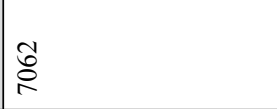 & 芯 & 占 & $\underline{n}$ \\
\hline 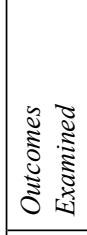 & 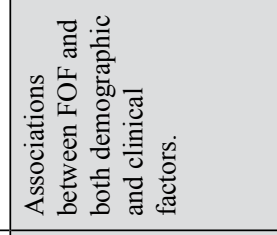 & 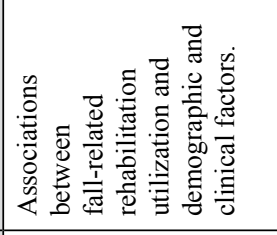 & 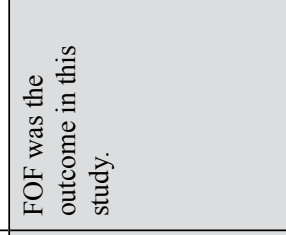 & 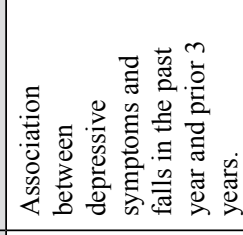 & 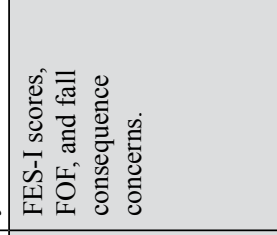 \\
\hline 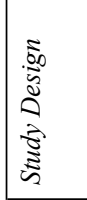 & 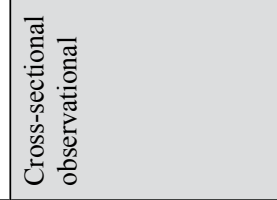 & 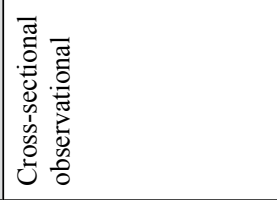 & 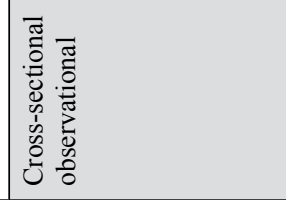 & 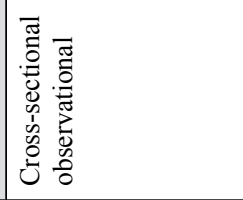 & 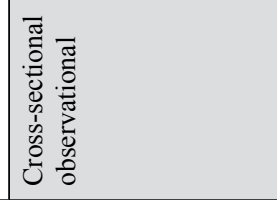 \\
\hline 蒿 & 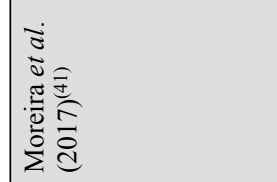 & 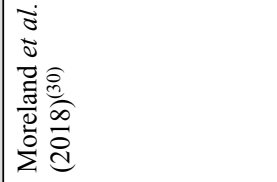 & 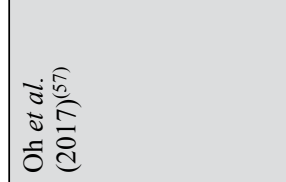 & 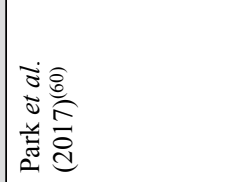 & 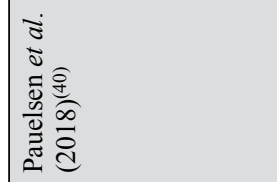 \\
\hline
\end{tabular}


MACKAY: FEAR OF FALLING IN OLDER ADULTS

\begin{tabular}{|c|c|c|c|c|c|c|c|}
\hline 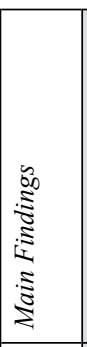 & 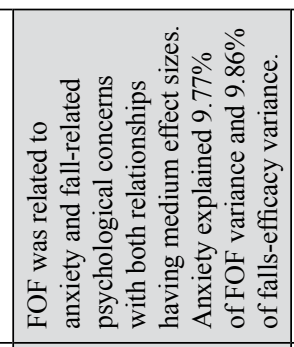 & 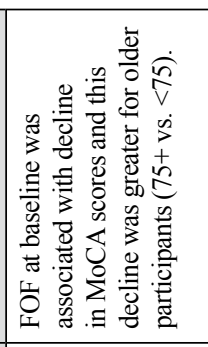 & 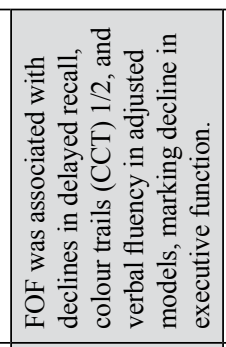 & 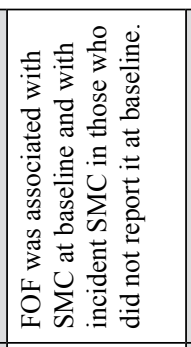 & 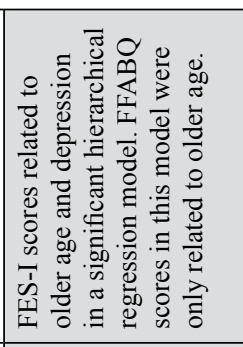 & 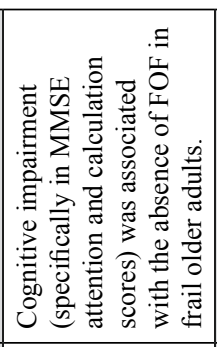 & 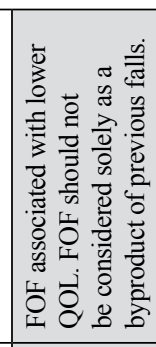 \\
\hline 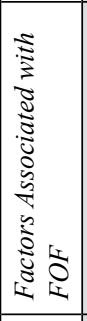 & 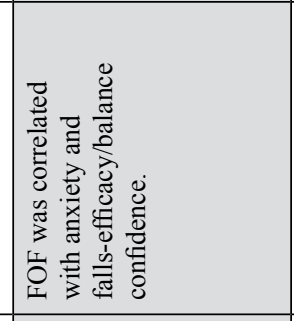 & 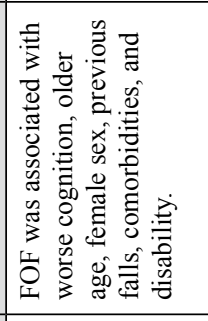 & 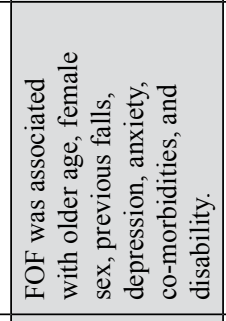 & 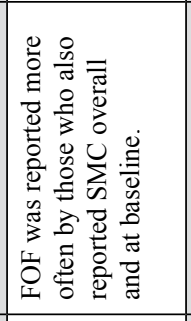 & 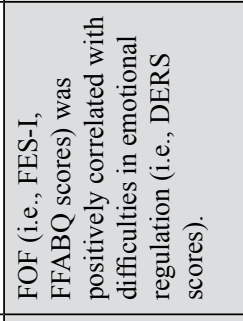 & 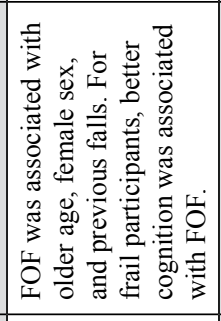 & 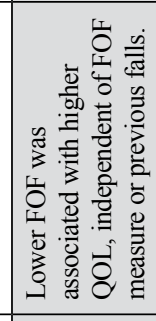 \\
\hline 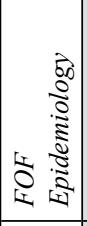 & $\sqrt{z}$ & 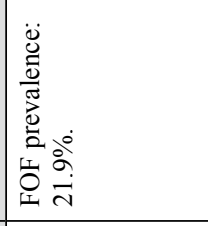 & 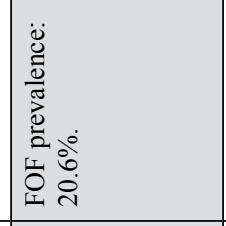 & 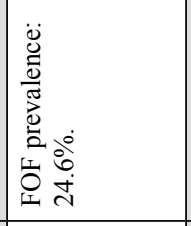 & 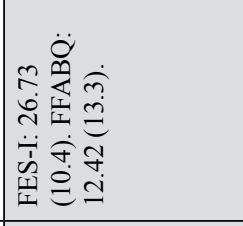 & 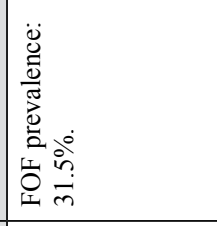 & $\overleftarrow{z}$ \\
\hline 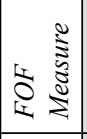 & 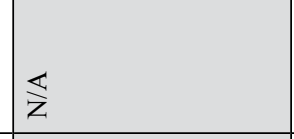 & 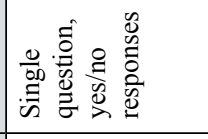 & 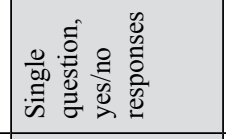 & 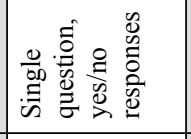 & 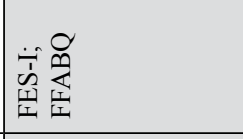 & 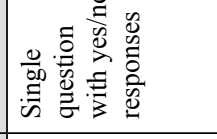 & $\overleftarrow{z}$ \\
\hline 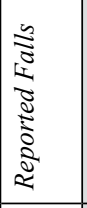 & $\mid \overleftarrow{z}$ & 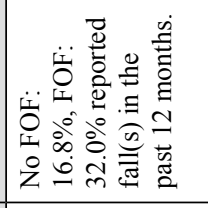 & 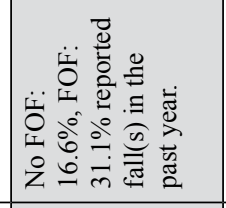 & $\mathbb{z}$ & 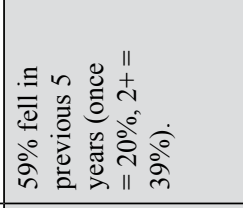 & 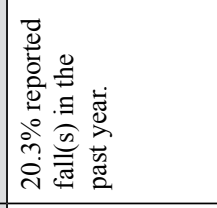 & $\overleftarrow{z}$ \\
\hline 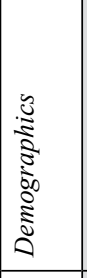 & 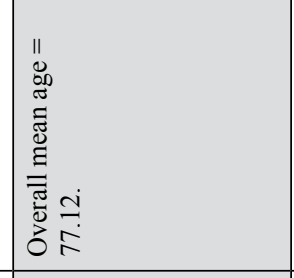 & 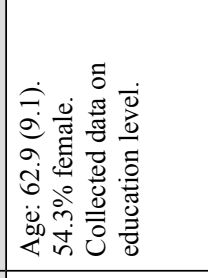 & 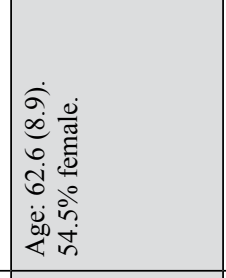 & 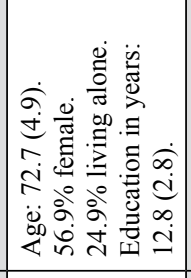 & 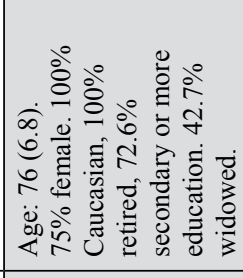 & 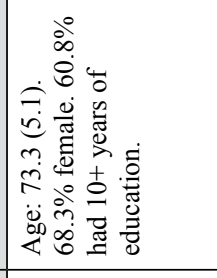 & 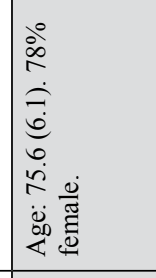 \\
\hline 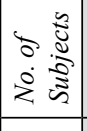 & $\begin{array}{l}\infty \\
\substack{f \\
f}\end{array}$ & $\overrightarrow{\widetilde{g}}$ & $\frac{\pi}{\frac{\pi}{n}}$ & \& & $\Xi$ & $\ddot{\mathscr{O}}$ & ठ্்ి \\
\hline 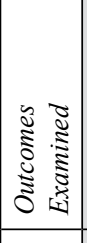 & 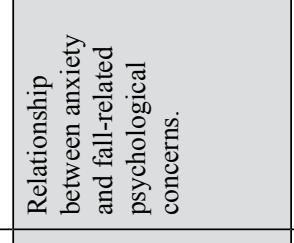 & 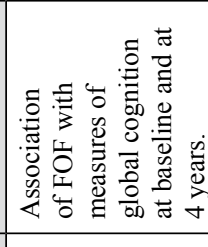 & 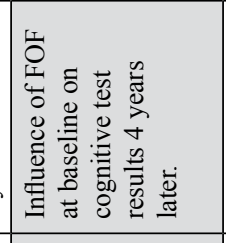 & 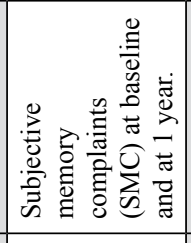 & 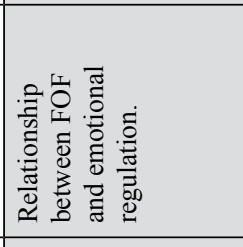 & 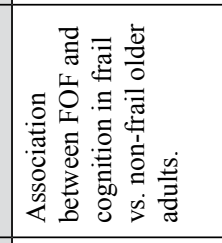 & 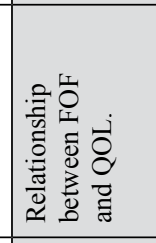 \\
\hline 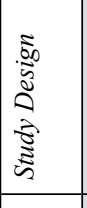 & 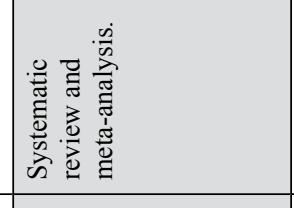 & 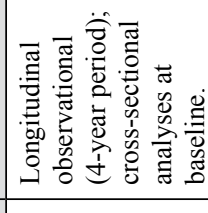 & 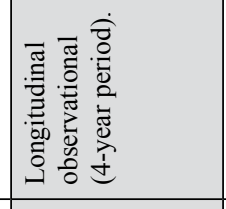 & 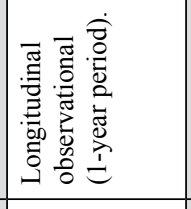 & 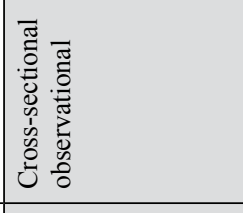 & 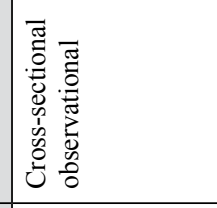 & 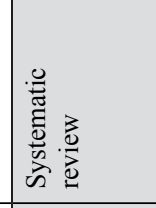 \\
\hline 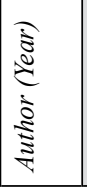 & 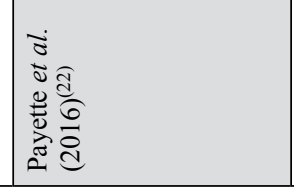 & 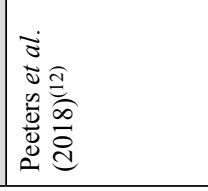 & 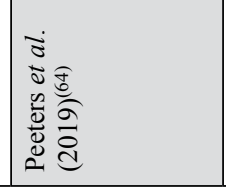 & 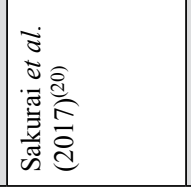 & 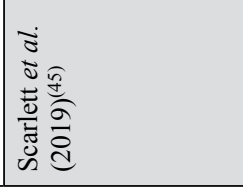 & 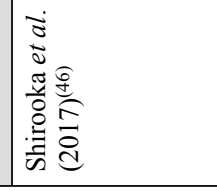 & 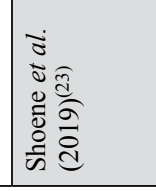 \\
\hline
\end{tabular}




\begin{tabular}{|c|c|c|c|c|c|c|}
\hline 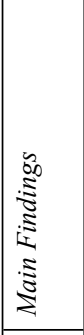 & 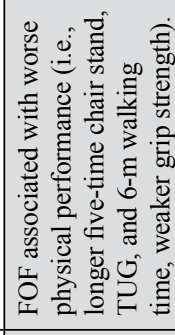 & 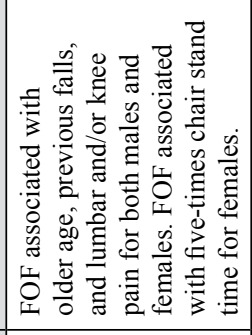 & 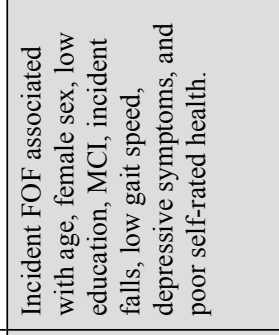 & 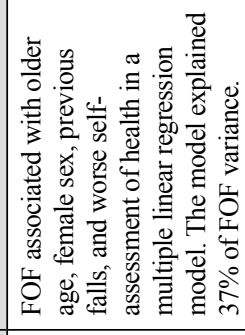 & 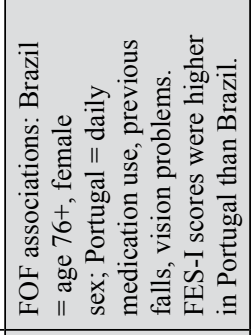 & \multirow{2}{*}{ 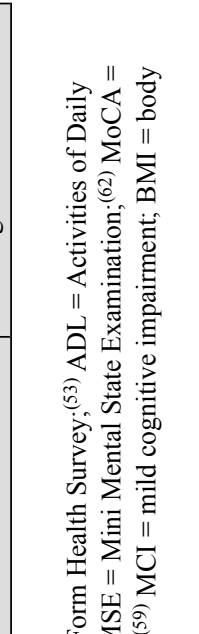 } \\
\hline 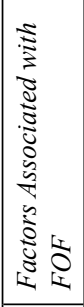 & 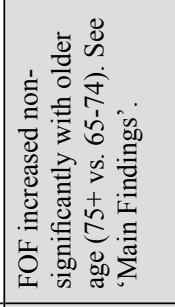 & 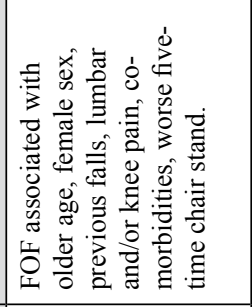 & 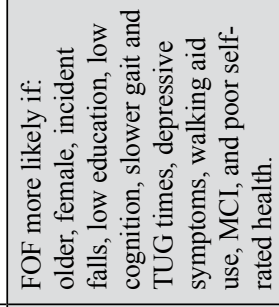 & 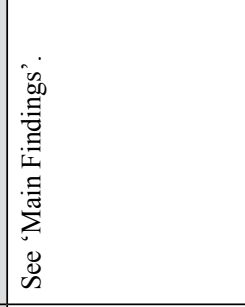 & 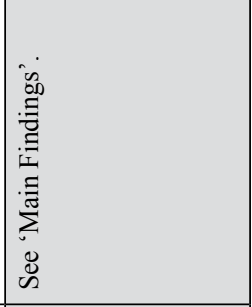 & \\
\hline 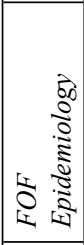 & 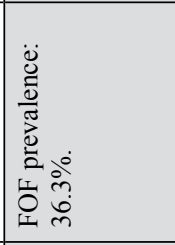 & 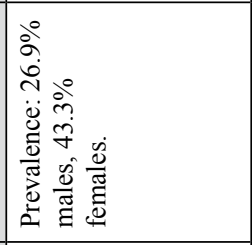 & 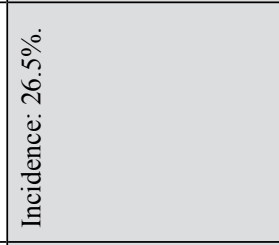 & 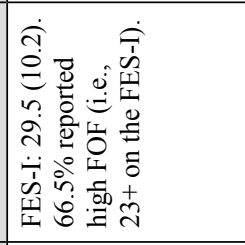 & 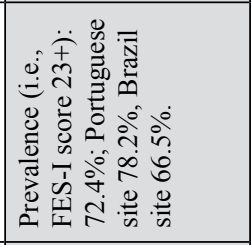 & 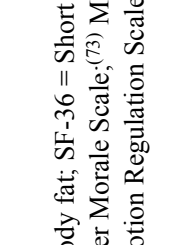 \\
\hline 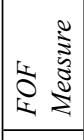 & 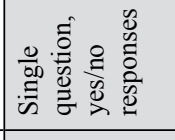 & 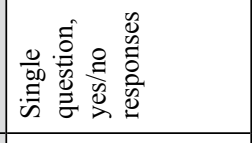 & 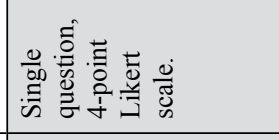 & \begin{tabular}{|l} 
\\
它 \\
至 \\
\end{tabular} & 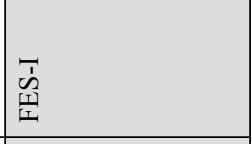 & 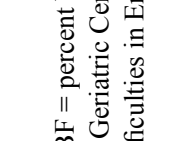 \\
\hline 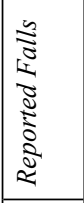 & 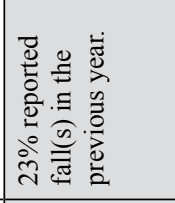 & 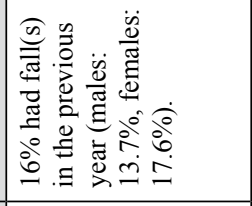 & 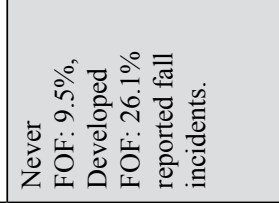 & 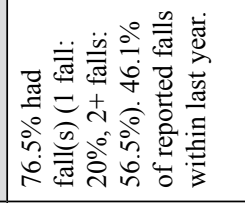 & 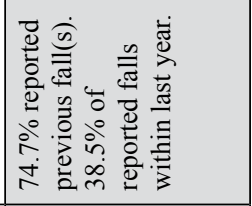 & 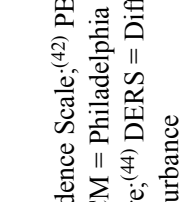 \\
\hline 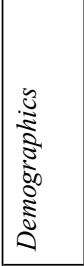 & 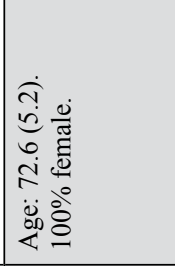 & 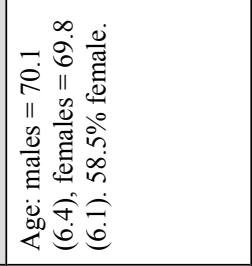 & 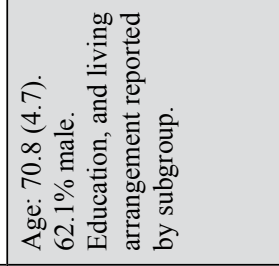 & 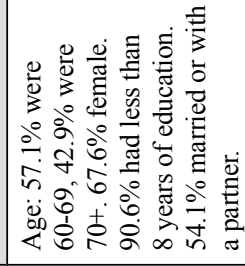 & 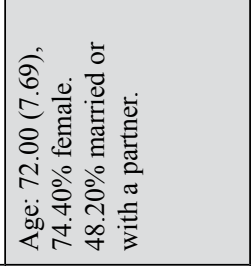 & 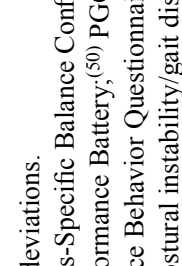 \\
\hline 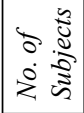 & $\stackrel{\infty}{\stackrel{\infty}{\imath}}$ & $\underset{\infty}{\mathbb{J}}$ & 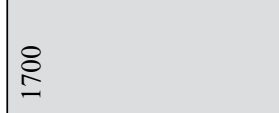 & $\cong$ & 商 & 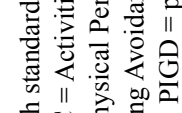 \\
\hline 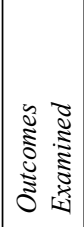 & 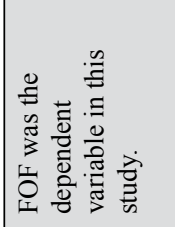 & 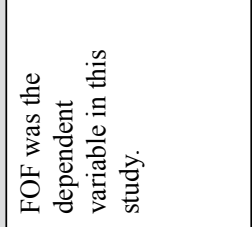 & 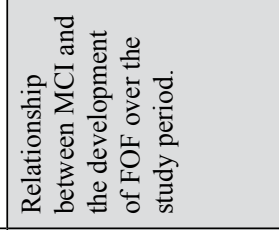 & 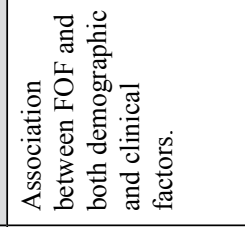 & 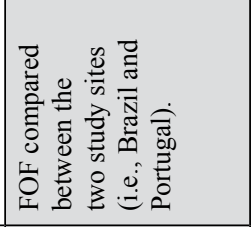 & 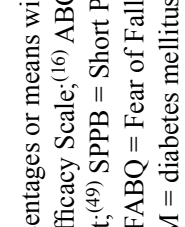 \\
\hline 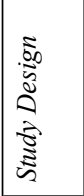 & 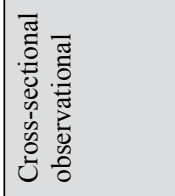 & 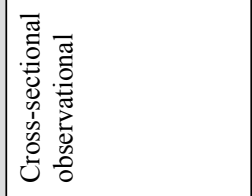 & 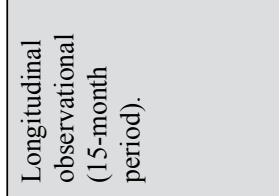 & 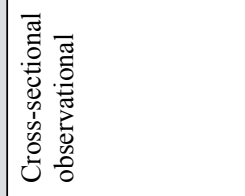 & 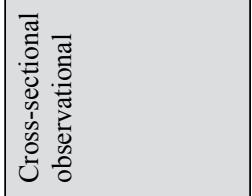 & 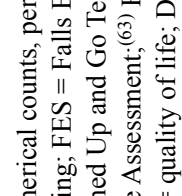 \\
\hline 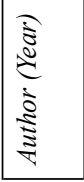 & 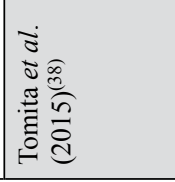 & 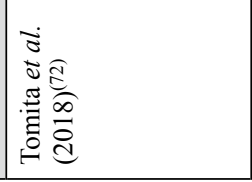 & 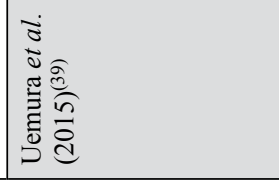 & 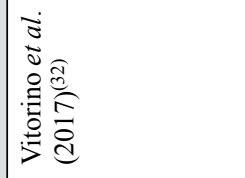 & 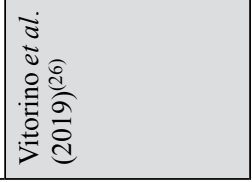 & 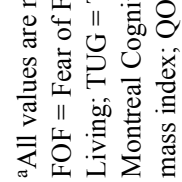 \\
\hline
\end{tabular}




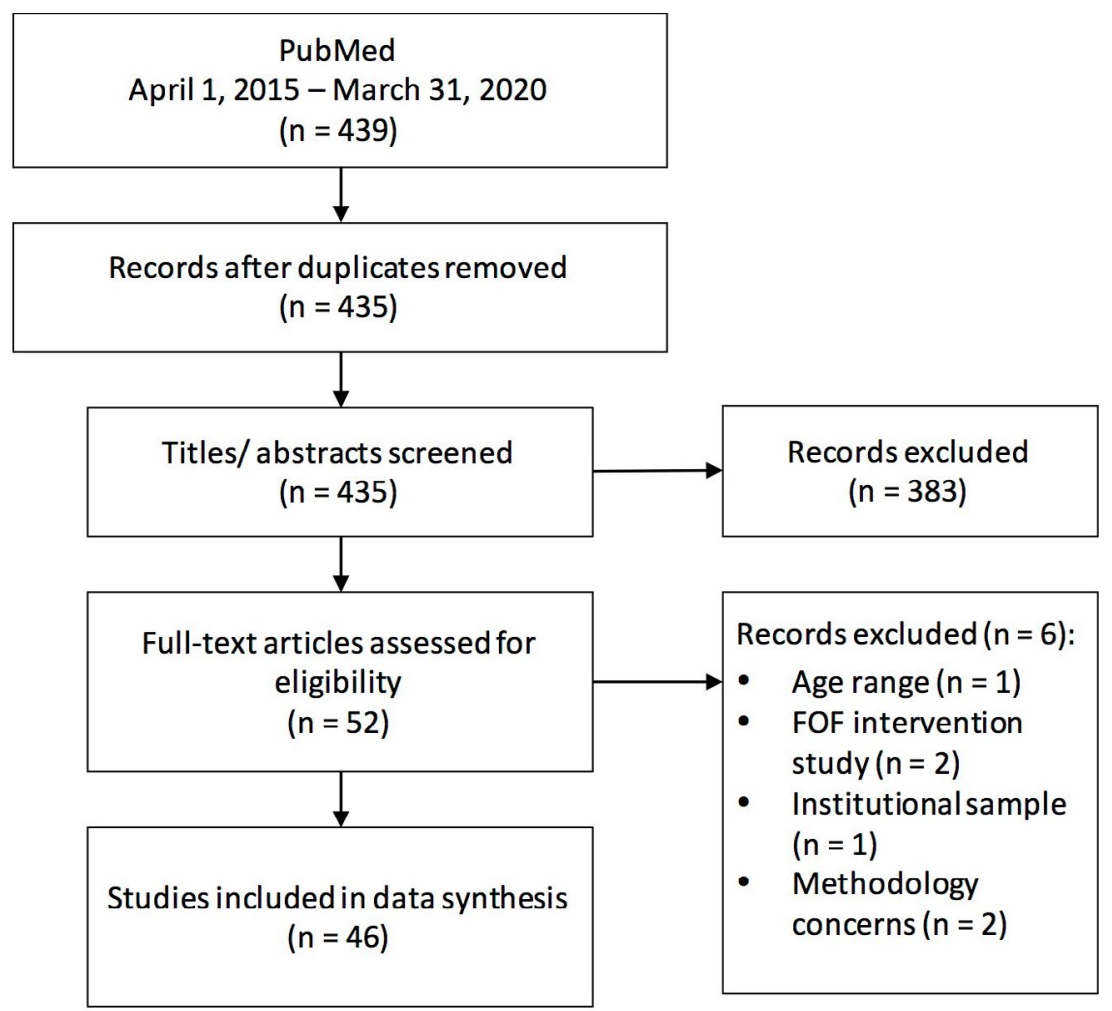

FIGURE 1. Flow diagram of article selection process

in studies done in Europe to $45.68 \%$ (SD 23.34) for those performed in Asia $(\mathrm{p}=.097)$.

\section{Sample Characteristics}

The 44 primary studies reviewed included a total of 124,841 participants. Sample sizes for these 44 studies ranged from 98 to 22,533 with a mean (SD) value of 3,448 (5643.8). When categorized by size, 10 studies had $<250$ participants, 12 had $250-1,000$, and 22 had $>1,000$ participants. Both systematic reviews ${ }^{(22,23)}$ included data from over 1,000 participants.

\section{Age}

An overall mean age could not be calculated as the required information was not provided in seven studies. ${ }^{(9,26-32)}$ Categorized by the mean age of participants, three of the studies predominately included participants aged 60-65, six with those aged 65-70, 16 with participants aged 70-75, 11 with those $75-80$, and three with participants $>80$.

\section{Biological Sex}

Most studies (38/46, 82.6\%) had predominantly ( $>50 \%)$ female participants, with three studies ${ }^{(33,34,38)}$ including only women. Six studies ${ }^{(8,14,21,28,36,38)}$ had predominantly male participants. Two studies ${ }^{(22,25)}$ did not report on the overall sex proportions of participants. The influence of gender identity or role was not examined.

\section{Fear of Falling Measurement}

Most primary studies $(29 / 44,65.9 \%)$ used a single question, which varied in wording and/or scoring, to detect FOF.
Nineteen studies used the wording "Are you afraid of falling?", while others specified a time period (e.g., "Have you worried about falling in the last four months?") or environment (e.g., "I am worried about falling when I walk in my neighborhood"). Ten of the studies that used a single question scored FOF on a Likert scale (e.g., never, rarely, sometimes, or often). Five studies ${ }^{(27,34,35,36,37)}$ asked about avoiding certain behaviours/activities as a result of FOF, and one inquired about perceived consequences of falling that could contribute to FOF. ${ }^{(40)}$

The Falls Efficacy Scale (FES) was used in 15 studies. Scores on this instrument range from 18-64 with higher scores representing greater FOF, ${ }^{(16)}$ though the thresholds used for defining the presence or severity of FOF varied between studies. For example, Auais et al. ${ }^{(13)}$ stated that FES scores of 16-19 indicated no/low FOF, while 20-27 denoted moderate and $\geq 28$ a high degree of FOF. Aibar-Almazan $e t$ $a l .{ }^{(33)}$ stated that values $>26$ placed an individual at higher risk of falling. Malini et al., ${ }^{(10)}$ de Souza Moreira et al., ${ }^{(41)}$ and Vitorino et al. ${ }^{(26,32)}$ all used a FES score cut-off of 23 to determine FOF. Vitorino et al. ${ }^{(26,32)}$ referred to these scores as indicating a high FOF.

The mean percent prevalence of FOF found in studies was significantly lower if a single question was used (mean \pm SD percentage $=37.38 \pm 19.53$ ) compared to those that used the FES (mean \pm SD percentage $=52.31 \pm 22.29 ; t=$ $-20.39, p<.05)$.

Other less commonly utilized instruments used to measure FOF included the Activities-Specific Balance 
Confidence Scale $^{(42)}(\mathrm{n}=3)$, Outdoor Fall Questionnaire ${ }^{(43)}(\mathrm{n}$ $=1$ ), Survey of Activities and Fear of Falling in the Elderly ${ }^{(15)}$ $(\mathrm{n}=1)$, and Fear Avoidance Behavior Questionnaire ${ }^{(44)}(\mathrm{n}=$ 1). Six studies ${ }^{(33,37,40,45,46,47)}$ used multiple approaches.

\section{Factors Associated with FOF}

Table 2 provides a summary of which studies found associations between FOF and the different categories of factors outlined below.

\section{Sociodemographics}

Female sex and older age were consistently associated with FOF. All 26 studies reporting on FOF and female sex found a positive association. Most studies (20/23, 87.0\%) reporting on age and FOF found a positive association (note: some dealt with age as a continuous variable while others used age categories). Having lower levels of education was associated with FOF in most studies $(8 / 13,61.5 \%)$ examining this relationship. Associations with other demographic variables such as living alone and/or having a low income, ${ }^{(9)}$ being a nondriver, ${ }^{(48)}$ and being widowed ${ }^{(10)}$ were noted in select studies.

\section{Physical Performance and Disability Measures}

Most studies (21/22, 95.5\%) reporting on FOF and physical performance and/or disability found positive associations between the presence of worse physical performance and/ or greater degrees of disability and FOF. The performance measures examined included the timed up-and-go (TUG), ${ }^{(45)}$ 6-metre walk time, chair stands, grip strength, gait speed, and Short Physical Performance Battery (SPPB). ${ }^{(50)}$ Disability measures included both basic and instrumental activities of daily living (e.g., Katz Index for ADLs, ${ }^{(51)}$ Lawton and Brody Scale for IADLs $\left.{ }^{(52)}\right)$. The Physical Functioning subscale of the 36-Item Short Form Survey (SF-36) ${ }^{(53)}$ was also used in three studies. ${ }^{(8,54,55)} \mathrm{In}$ all three worse scores were associated with FOF.

\section{Health Factors}

Comorbidity. All (24/24) studies examining this relationship found an association between medical morbidities and FOF. These studies either looked at the relationship with specific conditions (e.g., hypertension) or the presence of one or more morbidities. ${ }^{(56)}$ Obesity was found to be associated with FOF

TABLE 2.

Summary of Factors Associated with FOF

\begin{tabular}{|c|c|c|}
\hline Category & Associated Factor & Articles Reporting on Association (First Author Listed) \\
\hline \multirow[t]{3}{*}{ Socio-demographic } & Older Age & 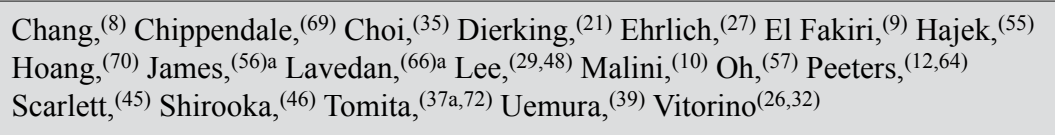 \\
\hline & Female Sex & $\begin{array}{l}\text { Auais, }{ }^{(13)} \text { Chang, }{ }^{(8)} \text { Chippendale, }{ }^{(69)} \text { Choi, }{ }^{(35)} \text { Dierking, }{ }^{(21)} \text { Ehrlich, }{ }^{(27)} \text { El Fakiri, }{ }^{(9)} \\
\text { Gazibara, }{ }^{(37)} \text { Hajek, }{ }^{(55)} \text { Hoang, }{ }^{(70)} \text { James, }{ }^{(56)} \text { Lavedan, }{ }^{(66)} \text { Lee, }{ }^{(29,48)} \text { Malini, }{ }^{(10)} \\
\text { Moreira, }{ }^{(41)} \text { Oh, }{ }^{(57)} \text { Park, }{ }^{\left({ }^{(60)}\right.} \text { Pauelsen, }{ }^{(40)} \text { Peeters, },{ }^{(12,64)} \text { Shirooka, }{ }^{(46)} \text { Tomita, }{ }^{(72)} \\
\text { Uemura, }{ }^{(39)} \text { Vitorino }{ }^{(26,32)}\end{array}$ \\
\hline & Lower Education & $\begin{array}{l}\text { Chang, }{ }^{(8) a} \text { Chippendale, }{ }^{(69) a} \text { Choi, }{ }^{(35)} \text { Dierking, }{ }^{(21) a} \text { El Fakiri, }{ }^{(9)} \text { Hoang, }{ }^{(70) a} \text { Lee, }{ }^{(48)} \\
\text { Malini, }{ }^{(10) a} \text { Moreira, }{ }^{(41)} \text { Oh, }{ }^{(57)} \text { Peeters, }{ }^{(12,64)} \text { Uemura }^{(39)}\end{array}$ \\
\hline Physical Performance & & $\begin{array}{l}\text { Aibar-Almazan, }{ }^{(33)} \text { Bjerk, }{ }^{(54)} \text { Chang },{ }^{(8)} \text { Chippendale, }{ }^{(69) a} \text { Dierking, }{ }^{(21)} \text { El Fakiri, }{ }^{(9)} \\
\text { Hajek, }{ }^{(55)} \text { Harada, }{ }^{(28)} \text { Johnson, }{ }^{(71)} \text { Lavedan, }{ }^{(66)} \text { Lee, }{ }^{(29)} \text { Makino, }{ }^{(67)} \text { Malini, }{ }^{(10)} \\
\text { Moreira, }{ }^{(41)} \text { Oh, }{ }^{(57)} \text { Park, }{ }^{(60)} \text { Pauelsen, }{ }^{(40)} \text { Peeters, }{ }^{(12,64)} \text { Tomita, },{ }^{(38,72)} \text { Uemura }^{(39)}\end{array}$ \\
\hline \multirow[t]{4}{*}{ Health } & Comorbidity & 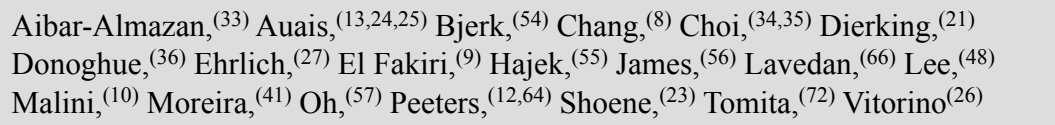 \\
\hline & Sensory Impairment & Choi, ${ }^{(34)}$ Ehrlich, ${ }^{(27)}$ Gazibara, ${ }^{(37)}$ James, ${ }^{(56)}$ Malini, ${ }^{(10)}$ Oh, ${ }^{(57)}$ Vitorino $^{(26)}$ \\
\hline & Falls & 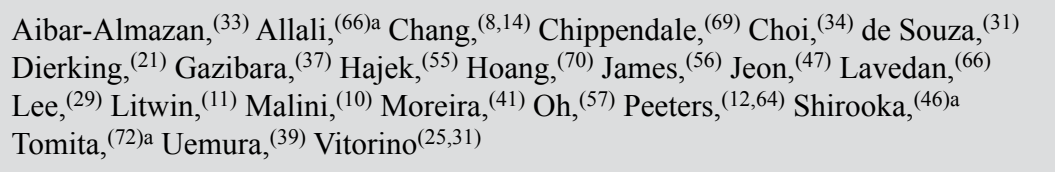 \\
\hline & Quality of Life & Bjerk, ${ }^{(54)}$ Chang, ${ }^{(8)}$ Shoene ${ }^{(23)}$ \\
\hline \multirow[t]{2}{*}{ Psychological } & Mood/Emotion & $\begin{array}{l}\text { Aibar-Almazan, }{ }^{(33)} \text { Bjerk, }{ }^{(54)} \text { Chang, }{ }^{(8)} \text { Chippendale, }{ }^{(69) a} \text { Choi, }{ }^{(34,35)} \text { Dierking, }{ }^{(21)} \text { El } \\
\text { Fakiri, }{ }^{(9)} \text { Hajek, }{ }^{(55)} \text { Hoang }{ }^{(70)} \text { Lavedan, }{ }^{(66)} \text { Lee, }{ }^{(48)} \text { Malini, }{ }^{(10)} \text { Moreira, }{ }^{(41)} \text { Park, }{ }^{(60)} \\
\text { Pauelsen, }{ }^{(40)} \text { Payette, }{ }^{(22)} \text { Peeters, }{ }^{(64)} \text { Scarlett, }{ }^{(45)} \text { Uemura }^{(39)}\end{array}$ \\
\hline & Cognition & $\begin{array}{l}\text { Choi, }{ }^{(34)} \text { Dierking, }{ }^{(21)} \text { Litwin, }{ }^{(11)} \text { Malini, }{ }^{(10)} \text { Moreira, } \\
\text { Uemura }^{(49)} \text { Peeters, }\end{array}$ \\
\hline $\begin{array}{l}\text { Social/ } \\
\text { Environmental }\end{array}$ & & Auais, ${ }^{(24)}$ Dierking, ${ }^{(21)}$ El Fakiri, ${ }^{(9)}$ Harada, ${ }^{(28)}$ Lee, ${ }^{(29,48)}$ Malini ${ }^{(10)}$ \\
\hline
\end{tabular}

${ }^{a}$ Non-significant association. 
by two studies ${ }^{(27,41)}$ and one study ${ }^{(33)}$ also found an association between FOF and sarcopenic obesity.

Sensory Impairment. Six studies $(10,26,27,37,56,57)$ found associations between sensory impairment (i.e., hearing, vision) and FOF. One study ${ }^{(34)}$ used a combined measure of sensory abilities and found an association between FOF and worse sensory function.

Falls. Most studies $(23 / 26,88.5 \%)$ providing data found an association between prior or incident falls and FOF.

Quality of Life. Two studies ${ }^{(8,54)}$ found an association between worse quality of life (QOL) measured by the SF-36 and FOF. One systematic review ${ }^{(23)}$ reported that FOF was associated with lower QOL regardless of the FOF measure used or whether there were prior falls.

\section{Psychological and Cognitive Status}

Psychological Status. Nearly all studies (19/20, 95.0\%) reporting on this found positive associations between FOF and the presence of depression and/or anxiety. The majority of these studies $(14 / 20,70.0 \%)$ examined the relationship with depressive symptoms measured by questionnaires (e.g., the Geriatric Depression Scale $\left.{ }^{(58)}\right)$. Scarlett et al. ${ }^{(45)}$ used the Difficulties in Emotional Regulation Scale (DERS) ${ }^{(59)}$ and found worse emotional regulation to be associated with FOF. One systematic review and meta-analysis ${ }^{(22)}$ found a significant, positive association between anxiety and both FOF and other fall-related concerns. Park et al ${ }^{(60)}$ found a positive association between FOF and anxiety as measured by the State-Trait Anxiety Inventory (STAI). ${ }^{(61)}$

Cognitive Status. Twenty-seven studies in our review examined cognition. In the majority of these studies $(18 / 27,66.7 \%)$ cognition was solely used for decisions on whether to include or exclude potential participants. The most commonly used measures were the Mini-Mental State Examination (MMSE) ${ }^{(62)}$ $(\mathrm{n}=18)$ and Montreal Cognitive Assessment $(\mathrm{MoCA})^{(63)}(\mathrm{n}=$ 3). In six studies, ${ }^{(10-12,34,39,64)}$ FOF was associated with worse cognition, while in two ${ }^{(21,46)}$ it was related to better cognition. One study ${ }^{(41)}$ found FOF to be associated with worse cognition only in participants who had diabetes mellitus.

Other Psychological Factors. Hajek et al. ${ }^{(55)}$ found that greater perceived stress, negative effect, and loneliness had positive associations with FOF, while higher levels of life satisfaction, positive effect, optimism, self-efficacy, selfesteem, and self-regulation showed negative associations. Oh et al. ${ }^{(57)}$ found lower life satisfaction to be associated with FOF. Pauelsen et al. ${ }^{(40)}$ found lower morale to be associated with FOF.

\section{Social and Environmental Relationships}

The associations found between social or environmental factors and FOF were generally limited to individual studies. Auais et al. ${ }^{(24)}$ found that FOF was associated with decreased life-space mobility. El Fakiri et al. ${ }^{(9)}$ reported that both living alone and in a deprived neighbourhood were associated with FOF. Harada et al. ${ }^{(28)}$ showed a relationship between FOF and rates of crime in one's neighbourhood. Lee et al. ${ }^{(29)}$ found that the presence of drainage ditches and broken sidewalks was associated with FOF, while lower traffic speeds were protective. Lee, Oh, and Hong ${ }^{(48)}$ reported that discomfort with one's neighbourhood was associated with FOF, while more social support and access to neighbourhood facilities were protective. Finally, Malini et al. ${ }^{(10)}$ showed that less social support was associated with FOF, while Dierking et al. ${ }^{(21)}$ found that frequent interaction with friends was protective.

\section{Outcomes Predicted by FOF}

Falls. Three prospective longitudinal studies $(11,31,65)$ found that baseline FOF predicted incident falls. Conversely, Lavedan et al. ${ }^{(66)}$ did not find FOF at baseline to significantly predict falls over a two-year study period.

Mortality. Chang et al. ${ }^{(14)}$ reported that FOF was associated with a higher risk of mortality over a seven-year period.

Functional Decline. Auais et al. ${ }^{(25)}$ found that FOF predicted incident mobility disability and the development of worse physical performance. Makino et al. ${ }^{(67)}$ showed that FOF predicted incident disability. Choi et al. ${ }^{(34)}$ demonstrated that FOF predicted greater functional decline.

Impaired Cognition. Peeters et al. ${ }^{(12)}$ found that FOF predicted greater decline in global cognition as measured by the MoCA. In a subsequent paper, Peeters et al. ${ }^{(64)}$ reported that cognitive decline associated with FOF was most evident on the Colour Trails Test (e.g., selective attention, executive functioning), delayed recall (i.e., memory), and verbal fluency (i.e., language, executive functioning). Sakurai et al..$^{(20)}$ found that FOF predicted incident subjective memory complaints.

\section{DISCUSSION}

Our scoping review confirmed that FOF is a highly prevalent issue associated with significant adverse outcomes in older adults and added a number of novel observations. The factors most consistently associated with the presence of FOF in this review were older age, female sex, previous falls, worse physical performance, and depressive symptoms. These findings are largely consistent with previous research. ${ }^{(7,18)}$ Many of the cross-sectional studies that identified these risk factors used multivariate regression models to identify the relative significance of individual factors or analyses stratified by sex ${ }^{(8,40)}$ or fall history. ${ }^{(29)}$ These analyses, while valuable, are limited by the cross-sectional methodology used, which restricts the conclusions that can be made about potential causality.

The longitudinal studies in our review reported that essentially the same risk factors for FOF as the crosssectional studies mentioned were operant, ${ }^{(21)}$ though specific associations with self-reported unsteadiness ${ }^{(36)}$ and mild cognitive impairment ${ }^{(39)}$ were also identified. These studies also reported that FOF predicted adverse outcomes such as future falls ${ }^{(11,31,65)}$ and declines in both physical ${ }^{(34,67)}$ and cognitive ${ }^{(12,64)}$ functioning, demonstrating the potential importance of identifying those at risk of FOF and intervening. 
Further longitudinal studies investigating FOF are needed for a better understanding of how best to detect FOF and to help develop effective prevention and intervention strategies.

Our review found that studies that measured FOF with a single question had significantly lower FOF prevalence rates than those using the FES. While single-question approaches with yes/no responses have demonstrated good test/retest reliability, ${ }^{(68)}$ they have been criticized for not being able to detect milder - though still clinically important — degrees of concern. This makes delineating the relationship between FOF and activity restriction less clear as a result. ${ }^{(7,17)}$ Some studies using a single question include a Likert scale for responses. (28) Less is known about the reliability of the responses obtained in this manner. The FES focuses on the person's self-efficacy or confidence in avoiding falls while doing various activities. This measure has similarly shown good test/retest reliability, ${ }^{(16)}$ and its continuous scoring allows for more nuanced results. However, the FES has been criticized for the use of different thresholds in operationally defining FOF, as we saw in our review, as well as the concern that fall self-efficacy does not fully map to the concept of FOF. (7) Previous studies that have compared FOF measures have determined that self-efficacy scales like the FES and ABC are more robust, sensitive measures of FOF. ${ }^{(17,65)}$ Our results are in agreement that the FES is likely more sensitive for detecting FOF than single questions, though further studies comparing the predictive validity of single questions to the FES and other FOF measurements are required.

The current review identified limitations in the FOF research done to date. Alongside the above mentioned relative lack of longitudinal studies, inconsistency in the measures used to detect FOF also presents a challenge in deriving conclusions from the existing literature. The time limits placed on measures of FOF and falls (e.g., "Have you worried about falling in the last four months?") also present challenges in comparing studies. Consensus on which approach to take in detecting FOF and the scoring used would help in addressing this. Other areas identified for future research would be the influence of gender (in contrast to biological sex) on FOF, and the relationship between FOF and cognition. For the latter, this remains under-investigated despite the known links between cognition and fall risk. Similarly, the relationship between FOF and its management with psychological, social, and environmental factors requires additional research. While the studies reviewed came from North and South America, Asia, and Europe (indicating that FOF is a worldwide issue), no recent papers were identified from either Africa or Australia. An unexplored area of inquiry would be critically searching for differences between regions and cultures in the prevalence, risk factors, and outcomes of FOF.

The current review has a number of limitations. We only examined studies conducted between April 2015 and March 2020, and our search was limited to the PubMed database. Studies that focused on FOF interventions or disease-specific populations were excluded. Our findings apply specifically to community-dwelling older adults. This review also did not include formalized quality assessments of the studies included for review, though study limitations, such as the identification of potential sources of bias, were included in the data extraction process.

\section{CONCLUSION}

FOF is common among community-dwelling older adults and is associated with adverse health outcomes. This scoping review provides a summary of recent observational studies on FOF that focused on factors associated with FOF, measurements used to quantify FOF, and gaps in the literature that could be addressed in future studies.

\section{ACKNOWLEDGEMENTS}

We would like to thank the Brenda Strafford Centre on Aging for their financial support in covering the article's processing fee.

\section{CONFLICT OF INTEREST DISCLOSURES}

The authors declare that no conflicts of interest exist.

\section{REFERENCES}

1. Bhala RP, O'Donnell J, Thoppil E. Ptophobia: phobic fear of falling and its clinical management. Phys Ther. 1982;62(2):187-90.

2. Tinetti ME, Powell L. Fear of falling and low self-efficacy: a cause of dependence in elderly persons. J Gerontol. 1993;48(Spec Issue):35-38.

3. Tinetti ME, Speechley M, Ginter SF. Risk factors for falls among elderly persons living in the community. $N$ England $J$ Med. 1988;319(26):1701-07.

4. Tideiksaar R. Falling in old age: Its prevention and treatment. New York: Springer Publishing Company; 1989.

5. Tideiksaar R. Falls in older people: Prevention and management. Baltimore, MD: Health Professions Press; 2002.

6. Whipple MO, Hamel AV, Talley KM. Fear of falling among community-dwelling older adults: a scoping review to identify effective evidence-based interventions. Geriatr Nurs. 2018; 39(2):170-77.

7. Jung D. Fear of falling in older adults: comprehensive review. Asian Nurs Res. 2008;2(4):214-22.

8. Chang HT, Chen HC, Chou P. Factors associated with fear of falling among community-dwelling older adults in the Shih-Pai study in Taiwan. PloS one. 2016;11(3):e0150612.

9. El Fakiri F, Kegel AA, Schouten GM, et al. Ethnic differences in fall risk among community-dwelling older people in the Netherlands. J Aging Health. 2018;30(3):365-85.

10. Malini FM, Lourenço RA, Lopes CS. Prevalence of fear of falling in older adults, and its associations with clinical, functional and psychosocial factors: the Frailty in Brazilian Older PeopleRio de Janeiro Study. Geriatr Gerontol Int. 2016;16(3):336-44.

11. Litwin H, Erlich B, Dunsky A. The complex association between fear of falling and mobility limitation in relation to late-life falls: a SHARE-based analysis. J Aging Health. 2018;30(6):987-1008. 
12. Peeters G, Leahy S, Kennelly S, et al. Is fear of falling associated with decline in global cognitive functioning in older adults: Findings from the Irish longitudinal study on ageing. $J \mathrm{Am} \mathrm{Med}$ Dir Assoc. 2018;19(3):248-54.

13. Auais M, Alvarado BE, Curcio CL, et al. Fear of falling as a risk factor of mobility disability in older people at five diverse sites of the IMIAS study. Arch Gerontol Geriatr. 2016;66:147-53.

14. Chang HT, Chen HC, Chou P. Fear of falling and mortality among community-dwelling older adults in the Shih-Pai study in Taiwan: a longitudinal follow-up study. Geriatr Gerontol Int. 2017;17(11):2216-23.

15. Lachman ME, Howland J, Tennstedt $\mathrm{S}$, et al. Fear of falling and activity restriction: the survey of activities and fear of falling in the elderly (SAFE). J Gerontol B Psychol Sci Soc Sci. 1998;53(1):P43-P50.

16. Tinetti ME, Richman D, Powell L. Falls efficacy as a measure of fear of falling. J Gerontol. 1990;45(6):P239-P243.

17. Greenberg SA. Analysis of measurement tools of fear of falling for high-risk, community-dwelling older adults. Clin Nurs Res. 2012;21(1):113-30.

18. Denkinger MD, Lukas A, Nikolaus $\mathrm{T}$, et al. Factors associated with fear of falling and associated activity restriction in community-dwelling older adults: a systematic review. $\mathrm{Am} J$ Geriatr Psychiatry. 2015;23(1):72-86.

19. Arksey H, O'Malley L. Scoping studies: towards a methodological framework. Int J Soc Res Methodol. 2005;8(1):19-32.

20. Sakurai R, Suzuki H, Ogawa S, et al. Fear of falling, but not gait impairment, predicts subjective memory complaints in cognitively intact older adults. Geriatr Gerontol Int. 2017; 17(7):1125-31.

21. Dierking L, Markides K, Al Snih S, et al. Fear of falling in older Mexican Americans: a longitudinal study of incidence and predictive factors. J Am Geriatr Soc. 2016;64(12):2560-65.

22. Payette MC, Belanger C, Léveillé V, et al. Fall-related psychological concerns and anxiety among community-dwelling older adults: Systematic review and meta-analysis. PLoS one. 2016;11(4): 0152848.

23. Schoene D, Heller C, Aung YN, et al. A systematic review on the influence of fear of falling on quality of life in older people: is there a role for falls? Clin Interv Aging. 2019;14:701.

24. Auais M, Alvarado B, Guerra R, et al. Fear of falling and its association with life-space mobility of older adults: a crosssectional analysis using data from five international sites. Age Ageing. 2017;46(3):459-65.

25. Auais M, French S, Alvarado B, et al. Fear of falling predicts incidence of functional disability 2 years later: a perspective from an international cohort study. J Gerontol A Biol Sci Med Sci. 2018;73(9):1212-25.

26. Vitorino LM, Marques-Vieira C, Low G, et al. Fear of falling among Brazilian and Portuguese older adults. Int J Older People Nurs. 2019;14(2):e12230.

27. Ehrlich JR, Hassan SE, Stagg BC. Prevalence of falls and fall-related outcomes in older adults with self-reported vision impairment. J Am Geriatr Soc. 2019;67(2):239-45.

28. Harada K, Park H, Lee S, et al. Joint association of neighborhood environment and fear of falling on physical activity among frail older adults. J Aging Phys Act. 2017;25(1):140-48.

29. Lee S, Lee C, Ory MG, et al. Fear of outdoor falling among community-dwelling middle-aged and older adults: the role of neighborhood environments. Gerontologist. 2018;58(6):1065-74.

30. Moreland BL, Durbin LL, Kasper JD, et al. Rehabilitation utilization for falls among community-dwelling older adults in the United States in the National Health and Aging Trends Study. Arch Phys Med Rehabil. 2018;99(8):1568-75.

31. de Souza AQ, Pegorari MS, Nascimento JS, et al. Incidence and predictive factors of falls in community-dwelling elderly: a longitudinal study. Cienc Saude Colet. 2019;24(9):3507-16.

32. Vitorino LM, Teixeira CA, Boas EL, et al. Fear of falling in older adults living at home: associated factors. Rev Esc Enferm USP. 2017;51.

33. Aibar-Almazán A, Martínez-Amat A, Cruz-Díaz D, et al. Sarcopenia and sarcopenic obesity in Spanish community-dwelling middle-aged and older women: association with balance confidence, fear of falling and fall risk. Maturitas. 2018;107:26-32.

34. Choi K, Jeon GS, Cho SI. Prospective study on the impact of fear of falling on functional decline among community dwelling elderly women. Int J Env Res Pub Health. 2017;14(5):469.

35. Choi K, Ko Y. Characteristics associated with fear of falling and activity restriction in South Korean older adults. $J$ Aging Health. 2015;27(6):1066-83.

36. Donoghue OA, Setti A, O'Leary N, et al. Self-reported unsteadiness predicts fear of falling, activity restriction, falls, and disability. J Am Med Dir Assoc. 2017;18(7):597-602.

37. Gazibara T, Kurtagic I, Kisic-Tepavcevic D, et al. Falls, risk factors and fear of falling among persons older than 65 years of age. Psychogeriatrics. 2017;17(4):215-23.

38. Tomita Y, Arima K, Kanagae M, et al. Association of physical performance and pain with fear of falling among communitydwelling Japanese women aged 65 years and older. Medicine. 2015;94(35).

39. Uemura K, Shimada H, Makizako H, et al. Effects of mild cognitive impairment on the development of fear of falling in older adults: A prospective cohort study. $\mathrm{J} \mathrm{Am} \mathrm{Med} \mathrm{Dir} \mathrm{Assoc.}$ 2015;16(12):1104.e9-1104.e13.

40. Pauelsen M, Nyberg L, Röijezon U, et al. Both psychological factors and physical performance are associated with fall-related concerns. Aging Clin Exp Res. 2018 Sep 1;30(9):1079-85.

41. de Souza Moreira B, Sampaio RF, Diz JB, et al. Factors associated with fear of falling in community-dwelling older adults with and without diabetes mellitus: findings from the Frailty in Brazilian Older People Study (FIBRA-BR). Exp Gerontol. 2017;89:103-11.

42. Powell LE, Myers AM. The activities-specific balance confidence (ABC) scale. J Gerontol A Biol Sci Med Sci. 1995;50(1):M28-M34.

43. Chippendale T, Knight R, An MS, et al. Development and validity of the outdoor falls questionnaire (OFQ). Am J Occup Ther. 2016;70(4 Suppl 1):7011500010p1.

44. Velozo CA, Peterson EW. Developing meaningful fear of falling measures for community dwelling elderly. Am J Phys Med Rehabil. 2001;80(9):662-73.

45. Scarlett L, Baikie E, Chan SW. Fear of falling and emotional regulation in older adults. Aging Ment Health. 2019;23(12): 1684-90.

46. Shirooka H, Nishiguchi S, Fukutani N, et al. Cognitive impairment is associated with the absence of fear of falling in community-dwelling frail older adults. Geriatr Gerontol Int. 2017;17(2):232-38.

47. Jeon M, Gu MO, Yim J. Comparison of walking, muscle strength, balance, and fear of falling between repeated fall group, one-time fall group, and nonfall group of the elderly receiving home care service. Asian Nurs Res. 2017;11(4):290-96. 


\section{MACKAY: FEAR OF FALLING IN OLDER ADULTS}

48. Lee S, Oh E, Hong GR. Comparison of factors associated with fear of falling between older adults with and without a fall history. Int J Env Res Pub Health. 2018;15(5):982.

49. Podsiadlo D, Richardson S. The timed "Up \& Go": a test of basic functional mobility for frail elderly persons. $J$ Am Geriatr Soc. 1991;39(2):142-48.

50. Guralnik JM, Simonsick EM, Ferrucci L, et al. A short physical performance battery assessing lower extremity function: association with self-reported disability and prediction of mortality and nursing home admission. J Gerontol. 1994;49(2):M85-M94.

51. Katz S, Downs TD, Cash HR, et al. Progress in development of the index of ADL. Gerontologist. 1970;10(1 Part 1):20-30.

52. Lawton MP, Brody EM. Assessment of older people: selfmaintaining and instrumental activities of daily living. Gerontologist. 1969;9(3 Part 1):179-86.

53. Stewart AL, Ware JE. Measuring functioning and well-being: the medical outcomes study approach. Durham, NC: Duke University Press; 1992.

54. Bjerk M, Brovold T, Skelton DA, et al. Associations between health-related quality of life, physical function and fear of falling in older fallers receiving home care. BMC Geriatr. 2018; 18(1):253.

55. Hajek A, Bock JO, König HH. Psychological correlates of fear of falling: Findings from the German Aging Survey. Geriatr Gerontol Int. 2018;18(3):396-406.

56. James EG, Conatser P, Karabulut M, et al. Mobility limitations and fear of falling in non-English speaking older MexicanAmericans. Ethnic Health. 2017;22(5):480-89.

57. Oh E, Hong GR, Lee S, et al. Fear of falling and its predictors among community-living older adults in Korea. Aging Ment Health. 2017;21(4):369-78.

58. Yesavage JA. Geriatric depression scale. Psychopharmacol Bull. 1988;24(4):709-11.

59. Gratz KL, Roemer L. Multidimensional assessment of emotion regulation and dysregulation: Development, factor structure, and initial validation of the difficulties in emotion regulation scale. J Psychopathol Behav Assess. 2004;26(1):41-54.

60. Park Y, Paik NJ, Kim KW, et al. Depressive symptoms, falls, and fear of falling in old Korean adults: the Korean Longitudinal Study on Health and Aging (KLoSHA). J Frailty Aging. 2017;6(3):144-47.

61. Spielberger CD. State-Trait anxiety inventory. The Corsini Encyclopedia Of Psychology. 2010 Jan 30:1.
62. Folstein MF, Robins LN, Helzer JE. The mini-mental state examination. Arch Gen Psychiatry. 1983;40(7):812.

63. Nasreddine ZS, Phillips NA, Bédirian V, et al. The Montreal Cognitive Assessment, MoCA: a brief screening tool for mild cognitive impairment. J Am Geriatr Soc. 2005;53(4):695-99.

64. Peeters G, Feeney J, Carey D, et al. Fear of falling: a manifestation of executive dysfunction? Int J Geriatr Psychiatry. 2019;34(8):1275-82.

65. Allali G, Ayers EI, Holtzer R, et al. The role of postural instability/gait difficulty and fear of falling in predicting falls in nondemented older adults. Arch Gerontol Geriatr. 2017;69:15-20.

66. Lavedán A, Viladrosa M, Jürschik $\mathrm{P}$, et al. Fear of falling in community-dwelling older adults: a cause of falls, a consequence, or both? PLoS one. 2018;13(3): $\mathrm{e} 0194967$.

67. Makino K, Makizako H, Doi T, et al. Impact of fear of falling and fall history on disability incidence among older adults: Prospective cohort study. Int J Geriatr Psychiatry. 2018;33(4): 658-62.

68. Oh-Park M, Xue X, Holtzer R, et al. Transient versus persistent fear of falling in community-dwelling older adults: incidence and risk factors. J Am Geriatr Soc. 2011:59(7):1225-31.

69. Chippendale T, Lee CD. Characteristics and fall experiences of older adults with and without fear of falling outdoors. Aging Ment Health. 2018;22(6):849-55.

70. Hoang OT, Jullamate P, Piphatvanitcha N, et al. Factors related to fear of falling among community-dwelling older adults. J Clin Nurs. 2017;26(1-2):68-76.

71. Johnson CS, McLeod KM. Relationship between fear of falling and perceived difficulty with grocery shopping. J Frailty Aging. 2017;6:33-36.

72. Tomita Y, Arima K, Tsujimoto R, et al. Prevalence of fear of falling and associated factors among Japanese communitydwelling older adults. Medicine. 2018;97(4).

73. Kent D, Kastenbaum R, Sherwood S, et al. Research planning and action for the elderly: the power and potential of social science. New York, NY: Behavioral Publications; 1972.

Correspondence to: David B. Hogan, MD, FCPSC, Division of Geriatric Medicine, Department of Medicine, Cumming School of Medicine, University of Calgary, HSC-3330 Hospital Drive NW, Calgary, AB T2N 4N1

E-Mail: dhogan@ucalgary.ca 